\title{
Synthesis of highly functionalized tetrahydropyridines with potential biological activity
}

\author{
Aleksandra Wasilewska, ${ }^{\text {a }}$ Franciszek Sączewski, ${ }^{a} *$ Maria Gdaniec, ${ }^{\text {b }}$ Anna Makowska, ${ }^{\text {a }}$ \\ and Patrick J. Bednarski ${ }^{c}$ \\ ${ }^{a}$ Department of Chemical Technology of Drugs, Medical University of Gdansk, \\ Al. Gen. J. Hallera 107, 80-416 Gdansk, Poland \\ ${ }^{b}$ Faculty of Chemistry, Adam Mickiewicz University, Grunwaldzka 6, 60-870 Poznan, Poland \\ ${ }^{c}$ Department of Pharmaceutical and Medicinal Chemistry, Institute of Pharmacy, \\ University of Greifswald, Friedrich Str. 17, 17487 Greifswald, Germany \\ E-mail: alwas@gumed.edu.pl, saczew@gumed.edu.pl
}

\begin{abstract}
It is found that 1,2-dihydropyridine derivatives: 6-aryl-2,3-dihydro-6a $H$-imidazo[1,2- $a$ ]pyrido$[1,2-c][1,3,5]$ triazin-5(6H)-ones and 3,6,7,8a-tetrahydro- $2 H$-diimidazo[1,2-c:1',2'-e $]$ pyrido[1,2-a][1,3,5]triazine underwent Diels-Alder reactions with highly reactive azadienophiles: 4phenyl-1,2,4-triazoline-3,5-dione and phthalazine-1,4-dione. The structures of the products were confirmed by IR, ${ }^{1} \mathrm{H}-{ }^{13} \mathrm{C}$ heterocorrelated 2D NMR spectra HSQC and HMBC, mass spectra as well as single crystal X-ray crystallography.
\end{abstract}

Keywords: 1,2-dihydropyridines, tetrahydropyridines, Diels-Alder reactions

\section{Introduction}

Imidazo[1,2-a]-1,3,5-triazines are of pronounced pharmacological interest as potent dihydrofolate reductase inhibitors, ${ }^{1} \mathrm{~A}_{1}$ adenosine receptor antagonists, ${ }^{2}$ antiviral agents ${ }^{3}$ and granulocyte colony-stimulating factor mimetics. ${ }^{4}$ They have been shown to affect the circulatory system $^{5}$ and thrombocyte aggregation ${ }^{6}$ as well as cause sedation and antinociception in mice. ${ }^{7}$ Their cytotoxicity toward human cancer cell lines and antioxidant properties have also been studied. $^{8}$

One of the synthetic routes toward this class of compounds consists of the reaction of 2chloro-4,5-dihydroimidazole 1 with pyridine alone ${ }^{9}$ or its mixture with a suitably substituted phenyl isocyanate ${ }^{10}$ as depicted in Scheme 1. 


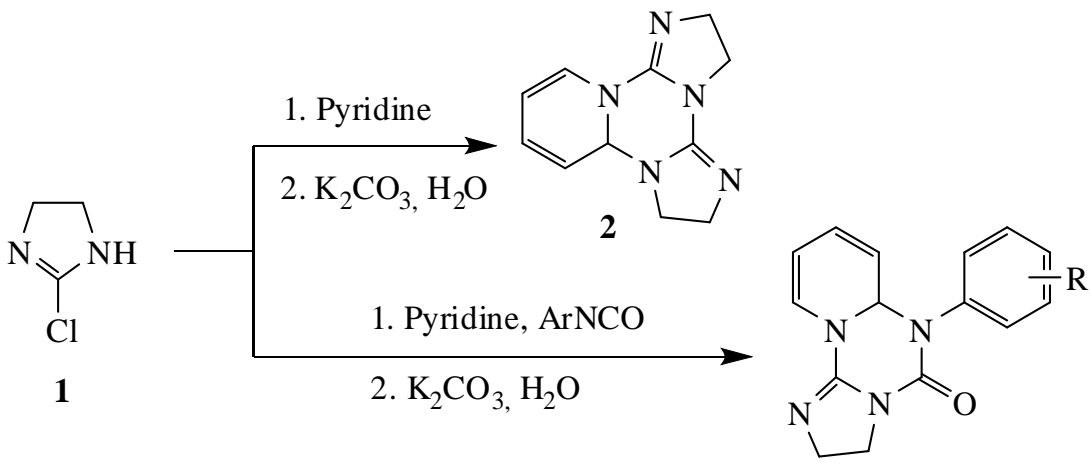

3

\section{Scheme 1}

The products 3,6,7,8a-tetrahydro-2H-diimidazo[1,2-c:1',2'-e]pyrido[1,2-a][1,3,5]triazine 2 or 6-aryl-2,3-dihydro-6a $H$-imidazo[1,2-a]pyrido[1,2-c][1,3,5]triazin-5(6H)-ones 3, respectively, contain a 1,2-dihydropyridine moiety that is expected to function as a diene and react (in a DielsAlder fashion) with suitably chosen dienophiles to give rise to new heteropolycyclic ring systems with potential biological activity. The [2+4] cycloaddition between carbon dienophiles and $\mathrm{N}$ protected 1,2-dihydropyridines, ${ }^{11} 2(1 H)$-pyridones, ${ }^{12}$ 2-methylene-1,2-dihydropyridines ${ }^{13}$ and other related systems is a well-established method of obtaining isoquinuclidine skeleton. The latter is present in natural products (iboga alkaloids, dioscorine) with central nervous system action $^{14}$ and hypoglycemic properties ${ }^{15}$ as well as various synthetic compounds of diverse pharmacological activity. ${ }^{16}$ The aim of the present studies was to test the utility of $\mathbf{2}$ and $\mathbf{3}$ as dienes for Diels-Alder reactions and to evaluate the cycloaddition products for cytotoxic activities on human cancer cell lines.

\section{Results and Discussion}

As shown in numerous reports ${ }^{11}$ on isoquinuclidine ring system synthesis 1,2-dihydropyridines are poorly reactive dienes, requiring elevated temperatures and long reaction times. These harsh reaction conditions seemed incompatible with compounds $\mathbf{2}$ or $\mathbf{3}$ that undergo rapid decomposition in solution at room temperature. This is especially true for $\mathbf{2}$, which is unstable even in the solid state and must be prepared just before use. The poor reactivity of simple 1,2dihydropyridine derivatives toward standard dienophiles may in some cases be overcome by the use of Lewis acid catalyst ${ }^{17}$ (which is intended to lower the LUMO energy of the dienophile ${ }^{18}$ ). This strategy was assumed inefficient with respect to compounds $\mathbf{2}$ and $\mathbf{3}$ because their binding with a Lewis acid would probably result in formation of even poorer dienes. Indeed, we found that addition of $\mathbf{2}$ or $\mathbf{3}$ into an equimolar mixture of $\mathrm{AlCl}_{3}$ and maleimide affords (after several hours) only the adducts of $\mathrm{AlCl}_{3}$ with 2 or 3 accompanied by decomposition products. Therefore 
another approach was needed and the use of highly reactive azadienophiles 4-phenyl-1,2,4triazoline-3,5-dione 4 and 1,4-phthalazinedione 5 seemed particularly appealing. There are quite a few reports on the reactions of 2-pyridones or $N$-protected 1,2-dihydropyridines with 4substituted 1,2,4-triazoline-3,5-diones ${ }^{19}$ or $\mathbf{5}^{19 \mathrm{c}}$ however, only in one case ${ }^{20}$ were pharmacological properties of the resultant cycloadducts, i.e. their CNS depressant and cardiotonic activities, seen.

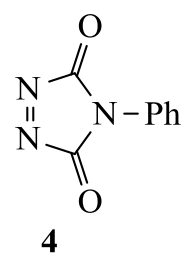<smiles>O=C1N=NC(=O)c2ccccc21</smiles>

The reactions of compounds 2 and 3 with 4-phenyl-1,2,4-triazoline-3,5-dione 4 were carried out at $-20{ }^{\circ} \mathrm{C}$ by dropwise addition of dichloromethane solution of $\mathbf{4}$ to diene dissolved in the same solvent. The consumption of the dienophile (indicated by discharge of its intense red color) was instantaneous and afforded complex reaction mixtures from which cycloadducts $\mathbf{6}-\mathbf{7 h}$ were isolated in 20-50\% overall yields by a combination of extraction and chromatographic methods (Scheme 2).

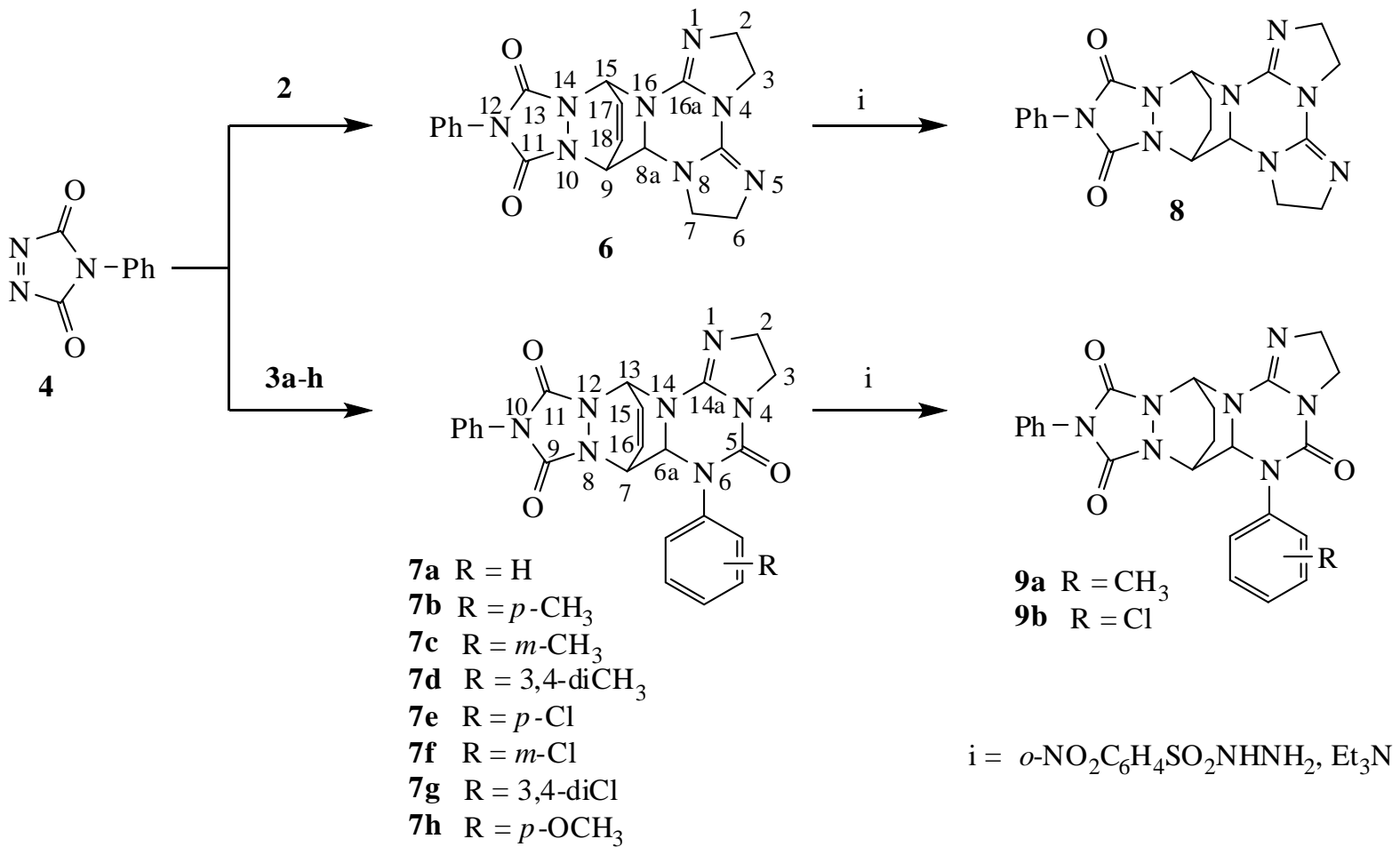

\section{Scheme 2}


The structures of the products were confirmed by ${ }^{1} \mathrm{H}$ NMR, ${ }^{13} \mathrm{C}$ NMR, IR and mass spectra as well as elemental analysis. The IR spectra of $\mathbf{6}$ and $\mathbf{7 a}-\mathbf{h}$ exhibited absorption at about 1780 and $1720 \mathrm{~cm}^{-1}$ characteristic for the stretching vibrations of 1,2,4-triazolidine-3,5-dione carbonyl groups and at about $1665 \mathrm{~cm}^{-1}$ corresponding to $\mathrm{C}=\mathrm{N}$ group. Absorptions from protons belonging to 2,3,5-triazabicyclo[2.2.2] oct-7-ene system were observed in the ${ }^{1} \mathrm{H}$ NMR spectra as a series of five well-resolved signals in the range of 4.5-7.2 ppm. Their assignment was based on ${ }^{1} \mathrm{H}-{ }^{13} \mathrm{C}$ heterocorrelated 2D NMR spectra HSQC and HMBC of $\mathbf{6}$ and 7e as representatives of the group (see supplementary material).

The two olefinic protons appeared as a pair of triplet-like multiplets localized at 6.59 and $6.99 \mathrm{ppm}$ in the spectrum of $\mathbf{6}$ and at about 6.5 and $7.2 \mathrm{ppm}$ in the case of compounds of type 7. The chemical shifts of doublets $(J \sim 5 \mathrm{~Hz})$ corresponding to the bridgehead protons: $15 \mathrm{H}$ in 6 and $13 \mathrm{H}$ in 7 were also very close, equal to 6.3 and $6.4 \mathrm{ppm}$, respectively. The substantial difference concerned the position of a $2 \mathrm{~Hz}$ doublet representing the proton of the $\mathrm{C}-\mathrm{N}$ bridge which appeared at $4.89 \mathrm{ppm}$ in the spectrum of $\mathbf{6}$ and at 5.8-6 ppm in the spectra of compounds $7 \mathbf{a}-\mathbf{h}$. The strong deshielding of proton H6a may be rationalized by the diamagnetic anisotropy of the neighboring phenyl ring. The same explanation should also account for the up-field shift of the bridgehead proton ( $\mathrm{H} 9$ or $\mathrm{H} 7$ for 6 or 7 respectively) from $5.45 \mathrm{ppm}$ in the spectrum of 6 to about 4.6-4.8 ppm in the spectra of $\mathbf{7 a}-\mathbf{h}$. As indicated by molecular models, $\mathrm{H} 7$ proton of $\mathbf{7}$ is placed above the phenyl ring and thus experiences the shielding effect of a diamagnetic ring current.

The ${ }^{13} \mathrm{C}$ NMR spectra of the products were also consistent with the proposed structures 6 and $7 \mathbf{a}-\mathbf{h}$. In the aliphatic region of $\mathbf{7 a}-\mathbf{h}$ spectra two signals of imidazoline carbon atoms $\mathrm{C} 3$ and $\mathrm{C} 2$ were found at about 45 and $51 \mathrm{ppm}$, respectively, followed by three resonance lines at 52, 62 and 67 ppm representing C7, C13 and C6a carbon atoms, respectively. The absorptions of the olefinic $\mathrm{C} 15$ and $\mathrm{C} 16$ atoms were observed at about 133 and $125 \mathrm{ppm}$. The two carbonyl groups of urazole moiety gave two distinct signals at 154 and 155 ppm, while $\mathrm{C} 5=\mathrm{O}$ and $\mathrm{C} 14 \mathrm{a}=\mathrm{N}$ carbons resonated at about 151 and $149 \mathrm{ppm}$, respectively.

An unexpected structure confirmation was provided by a mass spectrum of compound $\mathbf{7 e}$ that exhibited intense fragment ions at $m / z=227$ and $m / z=248$ (the latter accompanied by a freetimes less intense ion at $m / z=250$ ), arising from retro Diels-Alder dissociation of the undetectable molecular ion as depicted in Scheme 3. 


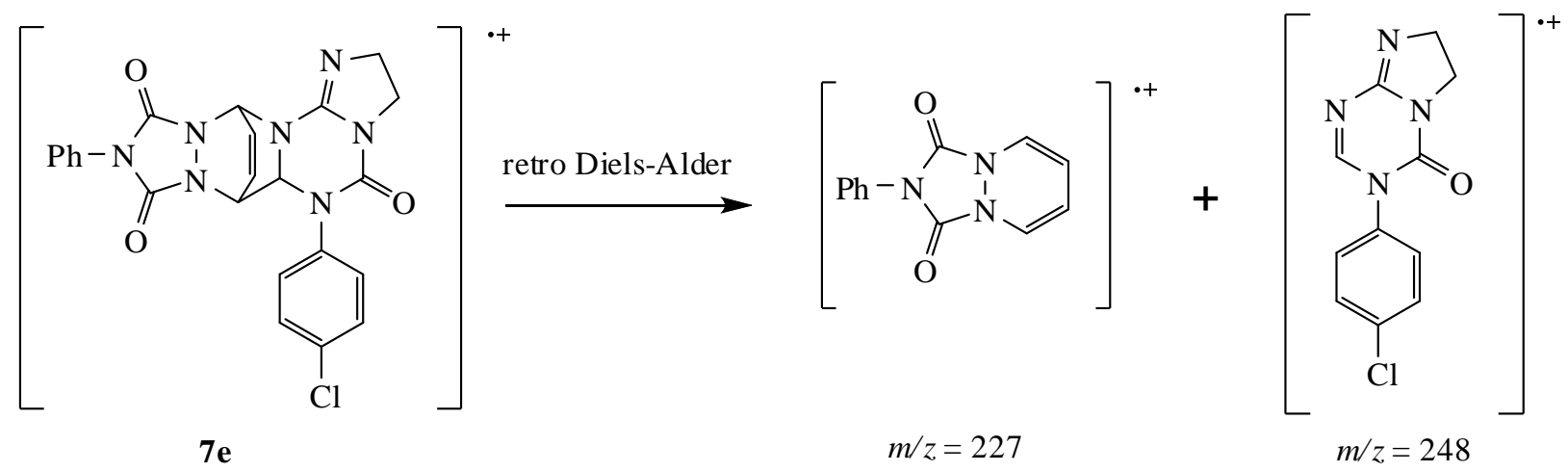

\section{Scheme 3}

Finally, good quality crystals of $\mathbf{6}$ were obtained from benzene that allowed unequivocal structure determination by X-ray diffraction methods (Figure 1). Compound $\mathbf{6}$ crystallizes in centrosymmetric space group $C 2 / c$ which means both enantiomers are present within the crystal lattice. The difficulties in obtaining efficient packing of large and irregularly shaped molecules of $\mathbf{6}$ are reflected by inclusion of the solvent (benzene and water) molecules into the crystal. The $\mathrm{X}$-ray structure analysis revealed formation of an endo product and indicated that the dienophile addition had taken place from the more hindered face of $\mathbf{2}$, that is the one containing H8a hydrogen atom. As a result, the $\mathrm{H} 8 \mathrm{a}$ atom is oriented exo with respect to the olefinic bridge.

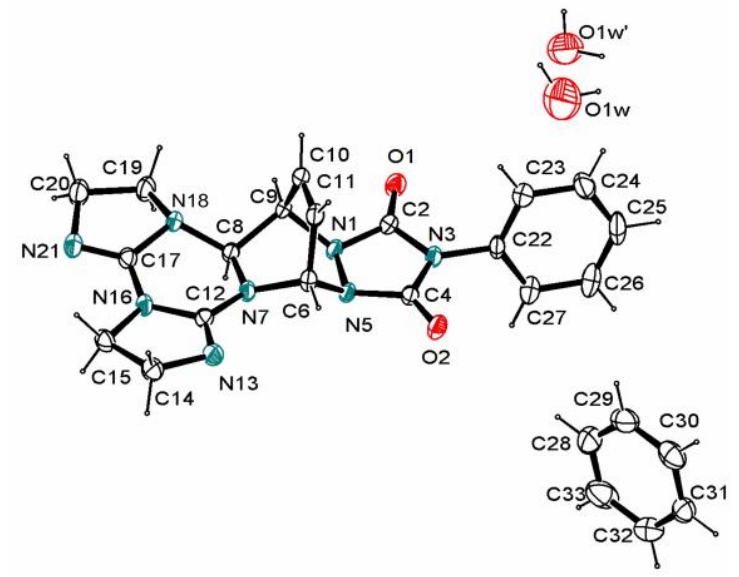

Figure 1. X-Ray structure of compound $\mathbf{6}$ showing atomic labels and displacement ellipsoids at the $50 \%$ probability level.

In the case of compounds $6,7 \mathbf{a}, 7 \mathbf{b}$ and $7 \mathrm{~h}$ only one stereoisomeric form of the cycloadduct was obtained whereas for $\mathbf{7} \mathbf{c}-\mathbf{g}$ (probably because of their higher chromatographic mobility) isolation of a minor amount of additional, more polar product became possible. The ${ }^{1} \mathrm{H}$ and ${ }^{13} \mathrm{C}$ NMR spectra of the two products shared the same basic features indicative for the presence of 2,3,5-triazabicyclo[2.2.2] oct-7-ene system, imidazoline moiety and two phenyl rings. Their mass spectra were identical, suggesting they were to each other stereoisomers. The most evident 
difference in the ${ }^{1} \mathrm{H}$ NMR spectra of the products concerned the absorption of proton H6a, which was observed at about $6 \mathrm{ppm}$ for the higher-yield stereoisomer while in the case of the loweryield one was shifted up-field to $5.4 \mathrm{ppm}$. According to literature reports, ${ }^{19 \mathrm{~b}, 21}$ the endo-protons belonging to bicyclo[2.2.2]oct-2-ene system and its aza counterparts are anisotropically shielded by the neighboring double bond. The above data allows one to conclude that the olefinic bridge and the H6a proton are oriented exo in the main product while endo in the minor one. To further explore this possibility the ROESY spectra of the two stereoisomers of compound 7e were taken (Figure 2). In the spectrum of the lower-yield product two diagnostic cross-peaks corresponding to H6a-H16 (stronger) and H6a-H15 (weaker) couplings were observed (Figure 2b). In the case of the main product no evidence for spatial vicinity of H6a to protons $\mathrm{H} 15$ or H16 was found (Figure 2a).
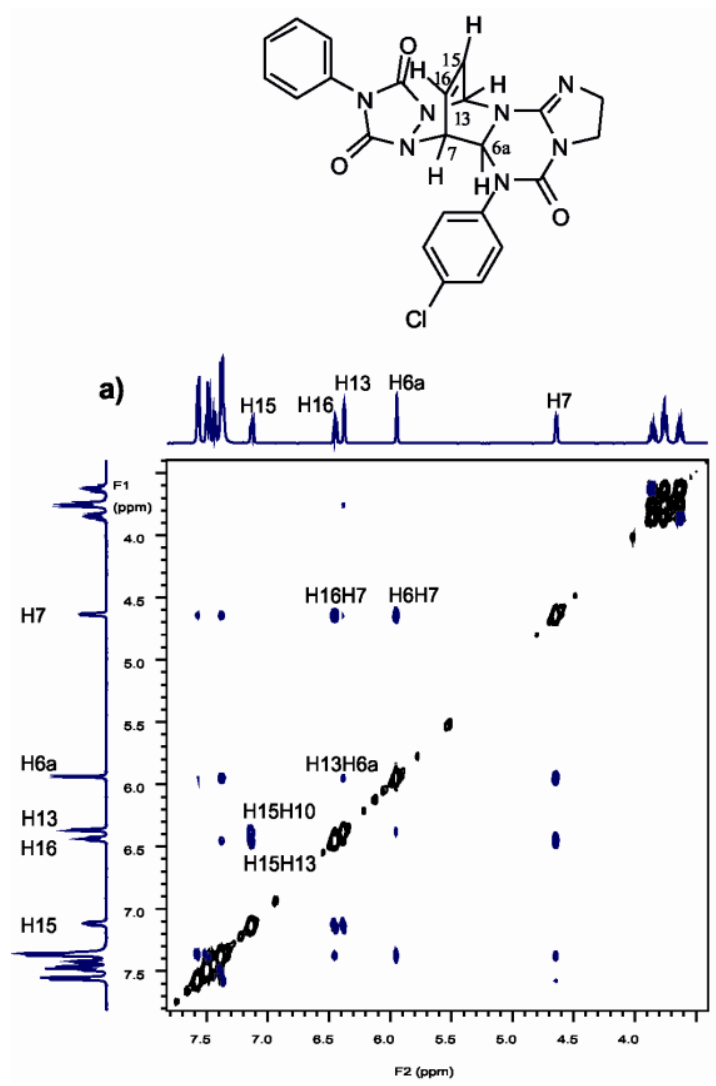
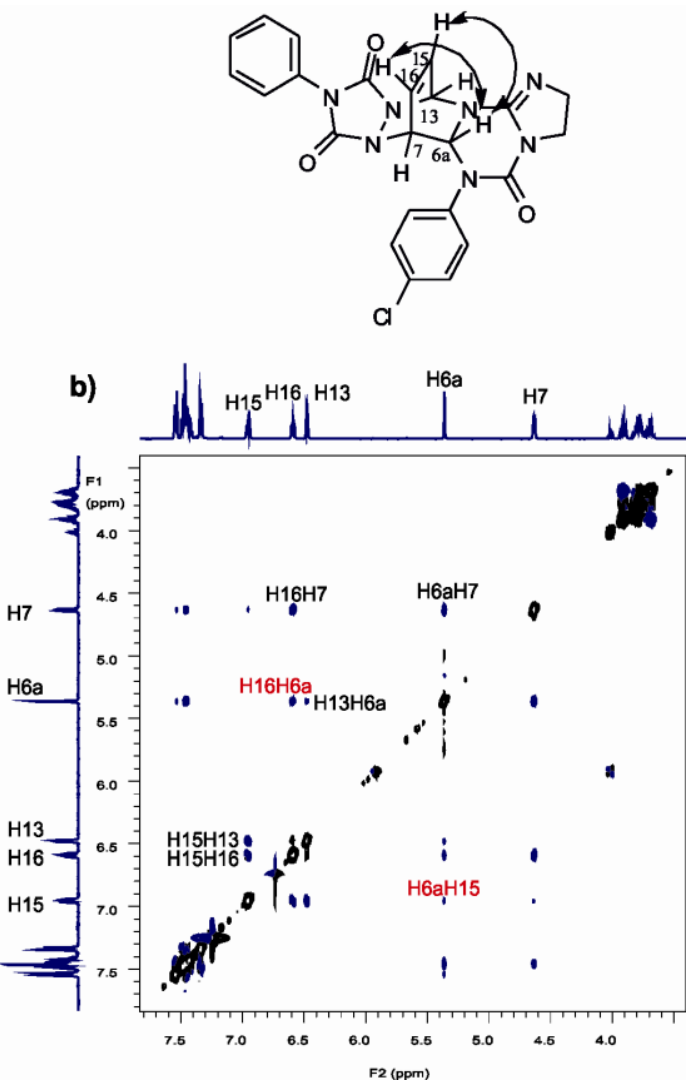

Figure 2. a) The ROESY spectrum and the proposed structure of the higher-yield stereoisomer of compound 7e. b) The ROESY spectrum and the proposed structure of the lower-yield stereoisomer of compound $\mathbf{7 e}$. The diagnostic cross-peaks are highlighted red.

In the next step reduction of the aliphatic double bond of compounds $6,7 \mathbf{b}$ and $7 \mathbf{e}$ as representatives of $\mathbf{6 - 7 h}$ series was attempted. Although catalytic (palladium on carbon) hydrogenation proved unsuccessful, the desired transformation was easily achieved in mild conditions using diimide generated in situ from $o$-nitrobenzenesulfonylhydrazide in the presence 
of triethylamine (Scheme 2). ${ }^{22}$ Upon reduction of the $\mathrm{C} 15=\mathrm{C} 16$ double bond the absorption of proton H6a was shifted down-field by $0.35 \mathrm{ppm}$ in the case of the lower-yield stereoisomer of 7e while remained unchanged for the higher-yield one. These observations are in agreement with the reported ${ }^{19 \mathrm{~b}, 21}$ influence of the olefinic bridge reduction on the chemical shifts of endo and exo protons belonging to bicyclo[2.2.2] oct-2-ene ring systems and further support our assignment of compounds' $7 \mathbf{a}-\mathbf{h}$ stereochemistry.

In contrast to their precursors, compounds 8, 9a and $\mathbf{9 b}$ formed good quality crystals, especially when recrystallized from acetonitrile. The reduced form of the higher-yield stereoisomer of $\mathbf{7 e}$ was obtained as a racemic compound, crystallizing in orthorombic space group $P b c a$. As evidenced earlier by ROESY experiments, the $\mathrm{C} 15=\mathrm{C} 16$ carbon bridge and H6a proton occupy the opposite sides of the molecule (Figure 3). Taking into account the stereochemistry of the transition state, the compound is exo. The twisting of the unsubstituted phenyl ring from the plane defined by urazole ring is substantially smaller than in the case of $\mathbf{6}$ which results in both $\mathrm{C}-\mathrm{H} \cdots \mathrm{O}=\mathrm{C}$ contacts of 2.310 and $2.452 \AA$ being shorter than the sum of the van der Waals radii of oxygen and hydrogen atoms $(1.52+1.2 \AA){ }^{23}$

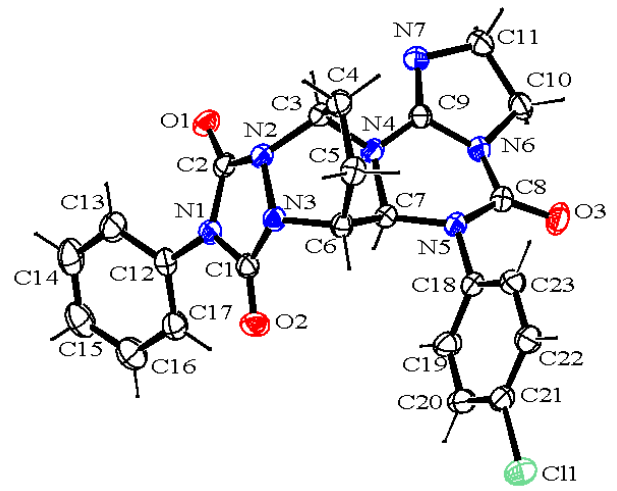

Figure 3. X-Ray structure of compound $\mathbf{9 b}$ showing atomic labels and displacement ellipsoids at the $50 \%$ probability level.

To further explore the utility of compounds $\mathbf{2}, \mathbf{3 a}-\mathbf{h}$ as dienes for Diels-Alder synthesis their reactions with phthalazine-1,4-dione $\mathbf{5}$ were studied. The green solution of $\mathbf{5}$ (obtained by vacuum filtration of the slurry resulting from oxidation of 2,3-dihydrophthalazine-1,4-dione 10 with lead tetraacetate in acetonitrile ${ }^{24}$ ) was added immediately to the cold solution of diene in $\mathrm{CH}_{2} \mathrm{Cl}_{2}$ and allowed to react (Scheme 4).

Chromatographic separation of thus obtained reaction mixtures afforded the desired cycloadducts only in the case of $\mathbf{3 b}$ and $\mathbf{3 g}$ while the other substrates gave intractable mixtures of products. When comparing the ${ }^{1} \mathrm{H}$ NMR spectra of adducts $11 \mathbf{a}$ and $\mathbf{1 1} \mathbf{b}$ with that of $\mathbf{7 a}-\mathbf{h}$ it can be seen that olefinic protons and the one belonging to $\mathrm{C}-\mathrm{N}$ bridge hold almost the same chemical shifts, while absorptions corresponding to both bridgehead protons of 11a and 11b are strongly (by about $1 \mathrm{ppm}$ ) shifted down-field to 5.54 and $7.23 \mathrm{ppm}$ for $\mathrm{H} 7$ and $\mathrm{H} 16$, respectively. Because of geometric reasons the bridgehead protons of $\mathbf{1 1 a}$ and $\mathbf{1 1 b}$ are more strongly influenced by 
diamagnetic anisotropy of carbonyl groups than their counterparts belonging to $7 \mathbf{a}-\mathbf{h}$ derivatives. The mass spectrum of 11a contains intense peaks at $m / z=212$ and $m / z=228$ that correspond to ions $\mathbf{A}$ and $\mathbf{B}$ resulting from retro Diels-Alder fragmentation of the molecular ion (Scheme 5). The above fragmentation pattern proves that $\mathbf{1 1 a}$ and $\mathbf{1 1 b}$ are indeed [4+2]cycloaddition products.

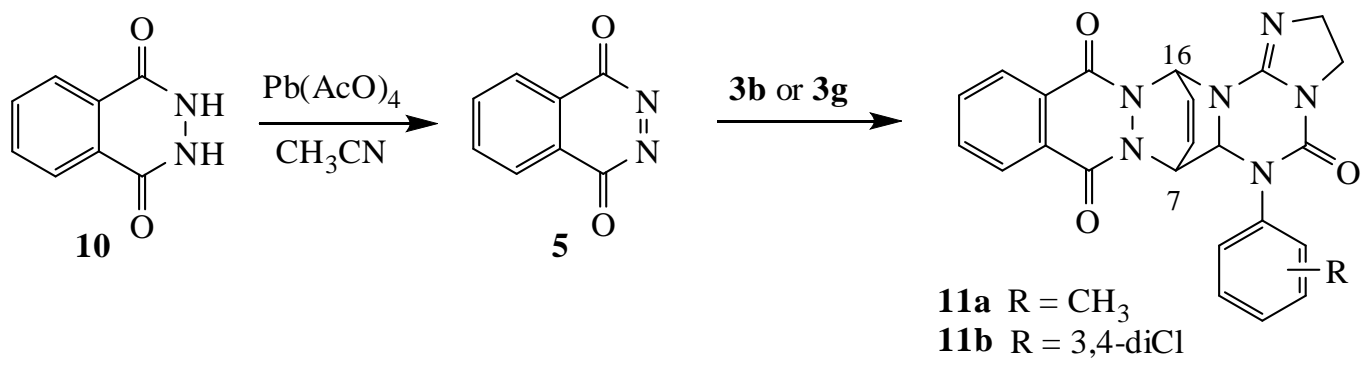

\section{Scheme 4}

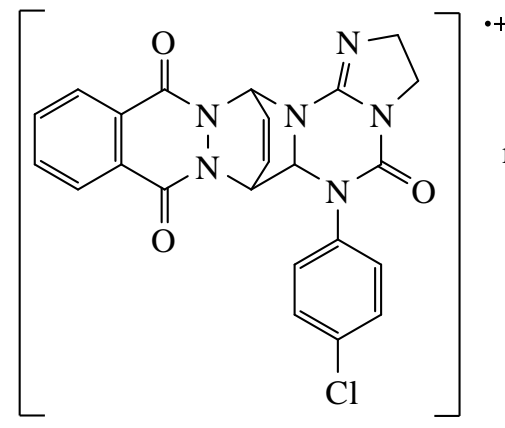

11a

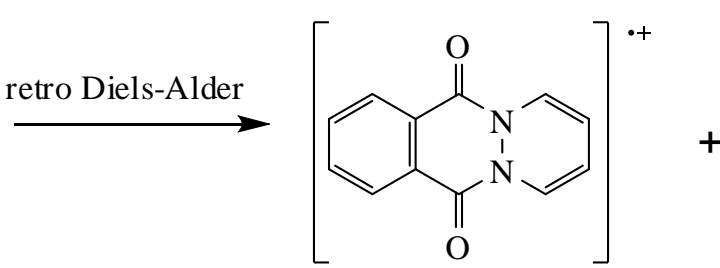

A $m / z=212$

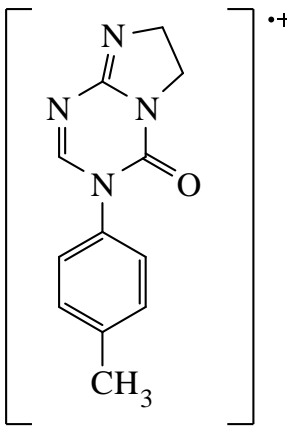

B $m / z=228$

\section{Scheme 5}

Table 1. $\mathrm{IC}_{50}$ values $(\mu \mathrm{M}) \pm \mathrm{S}$. D. in three human cancer cell lines as an average of 3-6 independent experiments

\begin{tabular}{cccc}
\hline Compound $^{\mathrm{a}}$ & \multicolumn{3}{c}{ Cell lines } \\
\cline { 2 - 4 } & LCLC-103H & A-427 & 5637 \\
\hline $\mathbf{6}$ & $14.73 \pm 2.70$ & $>20$ & $6.32 \pm 4.07$ \\
$\mathbf{7 e}$ & $14.86 \pm 2.37$ & $>20$ & $6.10 \pm 2.83$ \\
$\mathbf{7 e}$ & $9.61 \pm 2.55$ & $>20$ & $8.88 \pm 2.18$ \\
$\mathbf{7 g}$ & $15.82 \pm 0.68$ & $>20$ & $>20$ \\
$\mathbf{1 1 a}$ & $15.57 \pm 1.91$ & $>20$ & $16.49 \pm 1.28$ \\
$\mathbf{1 1 b}$ & $13.82 \pm 0.83$ & $10.51 \pm 0.83$ & $3.05 \pm 0.37$ \\
\hline
\end{tabular}

${ }^{\mathrm{a} C}$ Compounds that exhibited $\mathrm{IC}_{50}<20 \mu \mathrm{M}$ and were considered active. 7e' - The lower-yield stereoisomer of $\mathbf{7 e}$. 
Screening for cytotoxic activity of Diels-Alder adducts $\mathbf{6}, \mathbf{7 a}-\mathbf{h}, \mathbf{1 1 a}-\mathbf{b}$ and reduced derivatives 8, 9a-b was performed with three human cancer cell lines: the LCLC-103H large cell lung cancer, the A-427 small cell lung cancer and the 5637 bladder cancer. ${ }^{25}$ The results of the antiproliferation screen (Table 1) provide no clear conclusion about the structure activity relationship between phenyl ring substitution pattern of compounds $\mathbf{7 a - h}$ and their cytotoxicity. It seems that chlorine atoms, particularly in the para position, are preferred. This may suggest that lipophlicity of tested compounds plays important role.

It should be emphasized that all the reduced cycloadducts, including those originating from 6 and 7e, turned out to lack any antiproliferative activity. This indicates that the presence of the olefinic bridge is essential for the mechanism of action of compounds $6,7 e, 7 \mathbf{g}, 11 \mathrm{a}$ and $11 \mathbf{b}$. A possible explanation is that the double bond in question undergoes metabolic activation, for example epoxidation reaction, that affords derivatives able to alkylate nucleophilic targets within the tumor cell. This kind of metabolic activation has been proposed to explain the anti-cancer properties of acronycine and its derivatives containing $2 \mathrm{H}$-pyran ring. ${ }^{26}$

\section{Conclusions}

The use of highly reactive azadienophiles: 4-phenyl-1,2,4-triazoline-3,5-dione and 1,4phthalazinedione enabled us to obtain Diels-Alder cycloadducts of type 6, 7 and 11 from 1,2dihydropyridine derivatives of type 2 or 3 . Compounds $6,7 \mathbf{7}, 7 \mathbf{g}, \mathbf{1 1 a}$ and $\mathbf{1 1 b}$ derived from Diels-Alder reactions showed moderate cytotoxicity toward human cancer cell lines: 5637 and LCLC-103H. The $\mathrm{C}=\mathrm{C}$ double bond of 2,3,5-triazabicyclo[2.2.2] oct-7-en system present in the tested compounds appeared critical for their activity as its reduction afforded inactive derivatives.

\section{Experimental Section}

General. Melting points were measured on a Boëtius apparatus. The IR spectra were recorded in $\mathrm{KBr}$ pellets using Thermo Scientific Nicolet 380 FT-IR spectrometer. Nuclear magnetic resonance spectra were determined on Varian Gemini 200 or Varian Unity Plus 500 spectrometers. ${ }^{1} \mathrm{H}$ and ${ }^{13} \mathrm{C}$ chemical shifts were measured relative to the residual solvent signal at $7.26 \mathrm{ppm}$ and $77.0\left(\mathrm{CDCl}_{3}\right)$ or 2.50 and $39.5 \mathrm{ppm}$ (DMSO- $\left.d_{6}\right)$. Mass spectra were recorded on MAT95-Finnigan spectrometer operating at ionization potential of $70 \mathrm{eV}$. Phthalhydrazide 10 and phenyl isocyanates used in the studies were purchased from Alfa Aesar while 4-phenyl1,2,4-triazoline-3,5-dione 4, lead tetraacetate and 2-nitrobenzenesulfonyl chloride from Aldrich and used without further purification. 2-Chloro-4,5-dihydroimidazole hydrogen sulfate, ${ }^{27}$ 3,6,7,8a-Tetrahydro-2H-diimidazo[1,2-c:1',2'-e]pyrido[1,2-a][1,3,5]triazine $\quad \mathbf{2},{ }^{9} \quad$ 6-aryl-2,3dihydro-6aH-imidazo[1,2-a]pyrido[1,2-c][1,3,5]triazin-5(6H)-ones: ${ }^{10} \quad \mathbf{3 a}, \quad 3 \mathbf{3 e}, \quad 3 \mathbf{3 f}, \quad 3 \mathbf{h}, \quad o$ - 
nitrobenzenesulfonylhydrazide, ${ }^{28}$ phthalazine-1,4-dione $\mathbf{5}^{24}$ were prepared according to literature methods.

Flash column chromatography was performed by using 230-400 mesh silica gel 60 purchased from Alfa Aesar, while the plates used to perform preparative thin layer chromatography were coated with silica gel $60 \mathrm{PF}_{254}$ containing gypsum supplied by Merck. Preparative thin layer chromatography was performed using Chromatotron apparatus (Harrison Research Inc. USA).

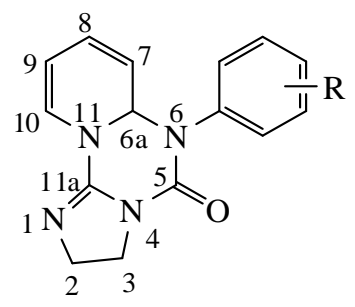

Figure 4. Numbering of atoms used to describe ${ }^{1} \mathrm{H}$ and ${ }^{13} \mathrm{C}$ NMR spectra of compounds $\mathbf{3 b}-\mathbf{d}$ and 3g.

Preparation of 6-aryl-2,3-dihydro-6a $H$-imidazo[1,2-a]pyrido[1,2-c][1,3,5]triazin-5(6H)ones: (3b-d) and (3g) was realized according to the procedure described in ref. 10 starting with $25 \mathrm{mmol}$ (2.5 g) of 1, $30 \mathrm{mmol}$ of an appropriate aromatic isocyanate and $75 \mathrm{mmol}(5.9 \mathrm{~g}, 6 \mathrm{ml})$ of pyridine.

6-(4-methylphenyl)-2,3-dihydro-6a $H$-imidazo[1,2- $a]$ pyrido $[1,2-c][1,3,5] \operatorname{triazin}-5(6 H)$-one (3b). Yellow crystals from ethanol/water, yield 42\%, $3 \mathrm{~g}, \mathrm{mp}(\mathrm{dec}): 163-164{ }^{\circ} \mathrm{C}$; IR $\left(v_{\max }, \mathrm{cm}^{-1}\right)$ : 3062, 3032, 2967, 2877, $1692(\mathrm{C}=\mathrm{O}), 1661(\mathrm{C}=\mathrm{N}), 1588,1466,1372,808 .{ }^{1} \mathrm{H}$ NMR (200 MHz, $\left.\mathrm{CDCl}_{3}\right): \delta_{\mathrm{H}} 2.36\left(3 \mathrm{H}, \mathrm{s}, \mathrm{CH}_{3}\right), 3.72-3.84(1 \mathrm{H}, \mathrm{m}, \mathrm{H} 3), 3.89-3.98(2 \mathrm{H}, \mathrm{m}, 2 \times \mathrm{H} 2), 4.08-4.22(1 \mathrm{H}$, m, H3'), 4.85 (1H, ddt, $J=10.2,3.2,1 \mathrm{~Hz}, \mathrm{H} 7), 5.14$ (1H, ddd, $J=7.6,5.9,1 \mathrm{~Hz}, \mathrm{H} 9), 5.96$ (1H, dddd, $J=10.2,5.9,1.7,1 \mathrm{~Hz}, \mathrm{H} 8), 6.45$ (1H, dd, $J=3.2,1.7 \mathrm{~Hz}, \mathrm{H6a}), 6.88$ (dt, $J=7.6,1$ $\mathrm{Hz}, \mathrm{H} 10), 7.06\left(2 \mathrm{H}, \mathrm{d}, J=8.4 \mathrm{~Hz}\right.$, aromat.), $7.20\left(2 \mathrm{H}, \mathrm{d}, J=8.4 \mathrm{~Hz}\right.$, aromat.). ${ }^{13} \mathrm{C} \mathrm{NMR}(50$ $\left.\mathrm{MHz}, \mathrm{CDCl}_{3}\right): \delta_{\mathrm{C}} 20.5\left(\mathrm{CH}_{3}\right), 44.7(\mathrm{C} 3), 51.5(\mathrm{C} 2), 67.9(\mathrm{C} 6 \mathrm{a}), 99.9(\mathrm{C} 9), 112.9(\mathrm{C} 7), 124.8$ (C8), 125.2 (C10), 129.6, 129.8, 132.5, 138.1 (6C aromat.), $151.4(\mathrm{C}=\mathrm{N}), 151.9(\mathrm{C}=\mathrm{O})$. Anal. Calcd for $\mathrm{C}_{16} \mathrm{H}_{16} \mathrm{~N}_{4} \mathrm{O}$ (280,32): C, 68.55; H, 5.75; N, 19.99\%. Found: C, 68.48; H, 5.68; N, $20.11 \%$

6-(3-methylphenyl)-2,3-dihydro-6aH-imidazo[1,2-a]pyrido[1,2-c][1,3,5]triazin-5(6H)-one (3c). Beige crystals from ethanol/water, yield 50\%, $3.5 \mathrm{~g}, \mathrm{mp}(\mathrm{dec}$.$) : 162-163{ }^{\circ} \mathrm{C}$; IR $\left(v_{\max }, \mathrm{cm}^{-1}\right)$ 3067, 3031, 2946, 2878, $1691(\mathrm{C}=\mathrm{O}), 1662(\mathrm{C}=\mathrm{N}), 1650,1587,1490,1373,1332,769 .{ }^{1} \mathrm{H}$ NMR $\left(200 \mathrm{MHz}, \mathrm{CDCl}_{3}\right): \delta_{\mathrm{H}} 2.35\left(3 \mathrm{H}, \mathrm{s}, \mathrm{CH}_{3}\right), 3.72-3.85(1 \mathrm{H}, \mathrm{m}, \mathrm{H} 3), 3.90-3.98(2 \mathrm{H}, \mathrm{m}, 2 \times \mathrm{H} 2)$, 4.08-4.21 (1H, m, H3'), 4.85 (1H, ddt, $J=10.2,3.2,1 \mathrm{~Hz}, \mathrm{H} 7), 5.15$ (1H, ddd, $J=7.6,5.9,1$ Hz, H9), 5.96 (1H, dddd, $J=10.2,5.9,1.7,1 \mathrm{~Hz}, \mathrm{H} 8), 6.46$ (1H, dd, $J=3.2,1.7 \mathrm{~Hz}, \mathrm{H6a}), 6.91$ (dt, $J=7.6,1 \mathrm{~Hz}, \mathrm{H} 10), 6.95-7.0$ (2H, m, aromat.), 7.13-7.17 (1H, m, aromat.), 7.24-7.32 (1H, m aromat.). ${ }^{13} \mathrm{C}$ NMR (50 MHz, $\left.\mathrm{CDCl}_{3}\right): \delta_{\mathrm{C}} 21.2\left(\mathrm{CH}_{3}\right), 44.7$ (C3), 51.5 (C2), 67.9 (H6a), 100.0 (C9), 112.9 (C7), 124.8 (C8), 125.2 (C10), 126.8, 128.9, 129.1, 130.5, 135.1, 139.2 (6C aromat.), 
$151.4(\mathrm{C}=\mathrm{N}), 152.1(\mathrm{C}=\mathrm{O})$. Anal. Calcd for $\mathrm{C}_{16} \mathrm{H}_{16} \mathrm{~N}_{4} \mathrm{O}(280,32)$ : C, 68.55; H, 5.75; N, $19.99 \%$. Found: C,68.67; H, 5.67; N, 19.88\%

\section{6-(3,4-dimethylphenyl)-2,3-dihydro-6a $H$-imidazo[1,2-a]pyrido[1,2-c $][1,3,5]$ triazin-5(6H)-}

one (3d). Beige crystals from ethanol/water, yield 30\%, $2.2 \mathrm{~g}, \mathrm{mp}(\mathrm{dec}):. 153-154{ }^{\circ} \mathrm{C}$; IR $\left(v_{\max }\right.$, $\left.\mathrm{cm}^{-1}\right):$ 3065, 3028, 2969, 2875, $1696(\mathrm{C}=\mathrm{O}), 1661(\mathrm{C}=\mathrm{N}), 1651,1587,1371,1331,772 .{ }^{1} \mathrm{H}$ NMR (200 MHz, $\left.\mathrm{CDCl}_{3}\right): \delta_{\mathrm{H}} 2.24\left(3 \mathrm{H}, \mathrm{s}, \mathrm{CH}_{3}\right), 2.25\left(3 \mathrm{H}, \mathrm{s}, \mathrm{CH}_{3}\right), 3.76-3.84(1 \mathrm{H}, \mathrm{m}, \mathrm{H} 3), 3.90-$ $3.98(2 \mathrm{H}, \mathrm{m}, 2 \times \mathrm{H} 2), 4.08-4.17(1 \mathrm{H}, \mathrm{m}, \mathrm{H} 3$ '), 4.87 (1H, ddt, $J=10.2,3,1 \mathrm{~Hz}, \mathrm{H} 7), 5.14(1 \mathrm{H}$, ddd, $J=7.6,5.9,1 \mathrm{~Hz}, \mathrm{H} 9), 5.95$ (1H, dddd, $J=10.2,5.9,1.7,1 \mathrm{~Hz}, \mathrm{H} 8), 6.44$ (1H, dd, $J=3$, $1.7 \mathrm{~Hz}, \mathrm{H6a}), 6.9$ (dd, $J=7.6,1 \mathrm{~Hz}, \mathrm{H} 10), 6.90$ (1H, d, $J=7.9 \mathrm{~Hz}$, aromat.), 6.96 (1H, s, aromat.), 7.15 (1H, d, $J=7.9 \mathrm{~Hz}$, aromat.); ${ }^{13} \mathrm{C} \mathrm{NMR}\left(50 \mathrm{MHz}, \mathrm{CDCl}_{3}\right): \delta_{\mathrm{C}} 19.4\left(\mathrm{CH}_{3}\right), 19.8$ $\left(\mathrm{CH}_{3}\right), 44.8$ (C3), $51.4(\mathrm{C} 2), 67.9$ (C6a), 100.1 (C9), 113.1 (C7), 124.8 (C8), 125.0 (C10), 127.0, 130.3, 129.8, 132.8, 136.9, $137.7(6 \mathrm{C}$, aromat.), $151.5(\mathrm{C}=\mathrm{N}), 152.0(\mathrm{C}=\mathrm{O})$. Anal. Calcd for $\mathrm{C}_{17} \mathrm{H}_{18} \mathrm{~N}_{4} \mathrm{O}$ (294,35): C, 69.37; H, 6.16; N, 19.03\%. Found: C, 69.28; H, 6.19; N, 19.09\%

6-(3,4-dichlorophenyl)-2,3-dihydro-6aH-imidazo[1,2- $a]$ pyrido[1,2-c] $[1,3,5]$ triazin-5(6H)one (3g). Yellow crystals ethanol/methanol/water, yield, $42 \%, 3.5 \mathrm{~g}, \mathrm{mp}$ (dec.): $176-177^{\circ} \mathrm{C}$; IR $\left(v_{\max }, \mathrm{cm}^{-1}\right): 3067,3032,2970,2878,1696(\mathrm{C}=\mathrm{O}), 1662(\mathrm{C}=\mathrm{N}), 1653,1588,1490,1374,1333$, 771. ${ }^{1} \mathrm{H}$ NMR (200 MHz, $\left.\mathrm{CDCl}_{3}\right): \delta_{\mathrm{H}} 3.71-3.84(1 \mathrm{H}, \mathrm{m}, \mathrm{H} 3), 3.91-4.00(2 \mathrm{H}, \mathrm{m}, 2 \times \mathrm{H} 2), 4.08-$ 4.21 (1H, m, H3'), 4.84 (1H, dd, $J=10.2,3.3 \mathrm{~Hz}, \mathrm{H} 7), 5.15-5.22$ (1H, m, H9), 6.02-6.10 (1H, m, H8), 6.49 (1H, dd, $J=3.3,1.7 \mathrm{~Hz}, \mathrm{H6a}), 6.88$ (d, $J=7.6, \mathrm{H} 10), 7.05$ (1H, dd, $J=8.5,2.4$ $\mathrm{Hz}$, aromat.), 7.32 (1H, d, $J=2.4 \mathrm{~Hz}$, aromat.), $7.47\left(1 \mathrm{H}, \mathrm{d}, J=8.5 \mathrm{~Hz}\right.$, aromat.). ${ }^{13} \mathrm{C} \mathrm{NMR}(50$ $\mathrm{MHz}, \mathrm{CDCl}_{3}$ ): $\delta_{\mathrm{C}} 44.7$ (C3), 51.6 (C2), 67.9 (C6a), 99.9 (C9), 112.0 (C7), 125.1 (C8), 126.3 (C10), 129.3, 130.6, 131.9, 132.5, 132.9, $134.4(6 \mathrm{C}$, aromat.), $151.0(\mathrm{C}=\mathrm{N}), 151.4(\mathrm{C}=\mathrm{O})$. Anal. Calcd for $\mathrm{C}_{15} \mathrm{H}_{12} \mathrm{C}_{12} \mathrm{~N}_{4} \mathrm{O}$ (335.19): C, 53.75; H, 3.61; N, 16.72\%. Found: C, 53.69; H, 3.53; N, $16.79 \%$.

\section{2-phenyl-2,3,6,7,8a,9,14,15-octahydro-9,15-ethenodiimidazo[1',2':1,2;1',2":5,6]}

\section{$[1,3,5]$ triazino[3,4- $d][1,2,4]$ triazolino[1,2-a][1,2,4]triazine-11(10H),13(12H)-dione (6)}

To a cooled $\left(-20{ }^{\circ} \mathrm{C}\right)$ and stirred solution of $2(0.56 \mathrm{~g}, 2.6 \mathrm{mmol})$ in $\mathrm{CH}_{2} \mathrm{Cl}_{2}(80 \mathrm{ml})$ a solution of 4-phenyl-1,2,4-triazoline-3,5-dione $(0.5 \mathrm{~g}, 2.86 \mathrm{mmol})$ in $\mathrm{CH}_{2} \mathrm{Cl}_{2}(50 \mathrm{ml})$ was added dropwise over 30 minutes under a nitrogen atmosphere. The stirring was continued for additional $30 \mathrm{~min}$. To the oil, obtained after evaporation of the reaction mixture, acetone $(30 \mathrm{ml})$ was added and on stirring beige solid precipitated. The acetone was decanted and the solid was extracted three times with equal portions of acetone $(\sim 20 \mathrm{ml})$. The combined acetone extracts were evaporated and the resulting solid was stirred for $1 \mathrm{~h}$ with water $(10 \mathrm{ml})$, filtered off, dried and purified through flash column chromatography using a mixture of dichloromethane, acetone and methanol $(3: 2: 0.5 \mathrm{v} / \mathrm{v} / \mathrm{v})$ as the eluent. Recrystallization from benzene gave colorless crystals of the product, yield $24 \%, 180 \mathrm{mg}, \mathrm{mp}$ (dec.): 228-230 ${ }^{\circ} \mathrm{C}$; IR $\left(v_{\max }, \mathrm{cm}^{-1}\right): 3436\left(\mathrm{H}_{2} \mathrm{O}\right), 3036$, 2958, 2883, $1779(\mathrm{C}=\mathrm{O}$ urazole $), 1724(\mathrm{C}=\mathrm{O}$ urazole $), 1656(\mathrm{C}=\mathrm{N}), 1647(\mathrm{C}=\mathrm{N}), 1496,1410$, 773, 733. ${ }^{1} \mathrm{H}$ NMR (200 MHz, DMSO-d $): \delta_{\mathrm{H}} 3.03-3.13$ (1H, m, H7), 3.29-3.45 (1H, m, H7'), 3.57-3.72 (6H, m, 2×H2, 2×H3, 2×H6), $4.89(1 \mathrm{H}, \mathrm{d}, J=2.5 \mathrm{~Hz}, \mathrm{H} 8 \mathrm{a}), 5.45(1 \mathrm{H}, \mathrm{dt}, J=5.9,2$ 
Hz, H9), 6.30 (1H, dd, $J=4.9,1.5 \mathrm{~Hz}, \mathrm{H} 15), 6.56-6.62(1 \mathrm{H}, \mathrm{m}, \mathrm{H} 18), 6.99$ (1H, ddd, $J=8.3$, 4.9, $1.5 \mathrm{~Hz}, \mathrm{H} 17), 7.39-7.57$ (5H, m, Ph). ${ }^{13} \mathrm{C}$ NMR (50 MHz, DMSO- $\left.d_{6}\right): \delta_{\mathrm{C}} 46.1$ (C3), 48.8 (C7), 50.9 (C6), 51.1 (C2), 52.7 (C9), 62.0 (C15), 68.2 (C8a), 126.3 (2C aromat.), 126.8 (C18), 128.9, 129.2, 130.7 (4C aromat.), $131.5(\mathrm{C} 17), 149.6(\mathrm{C} 17 \mathrm{a}=\mathrm{N}), 153.62(\mathrm{C} 4 \mathrm{a}=\mathrm{N}), 154.2,155.5$ (2×C $=\mathrm{O}$, urazole). Anal. Calcd for $\mathrm{C}_{19} \mathrm{H}_{18} \mathrm{~N}_{8} \mathrm{O}_{2} \cdot \mathrm{C}_{6} \mathrm{H}_{6} \cdot \mathrm{H}_{2} \mathrm{O}$ (486.53): C, 61.72; H, 5.39; N, $23.03 \%$. Found: $\mathrm{C}, 61.65 ; \mathrm{H}, 5.20 ; \mathrm{N}, 22.89 \%$

General procedure of the preparation of 6-aryl-2,3,6a,7,12,13-hexahydro-7,13ethenoimidazo[1',2':1,2][1,3,5] triazino[3,4-d][1,2,4]triazolino[1,2-a][1,2,4]triazine$6(5 H), 9(8 H), 11(10 H)$-triones $(7 a-h)$

To a cooled $\left(-20{ }^{\circ} \mathrm{C}\right)$ and stirred solution of the appropriate 6-aryl-2,3-dihydro-6a $H$-imidazo[1,2a]pyrido[1,2-c][1,3,5]triazin-5(6H)-one $(2.6 \mathrm{mmol})$ in $\mathrm{CH}_{2} \mathrm{Cl}_{2}(80 \mathrm{ml})$ a solution of 4-phenyl1,2,4-triazoline-3,5-dione $(0.5 \mathrm{~g}, 2.86 \mathrm{mmol})$ in $\mathrm{CH}_{2} \mathrm{Cl}_{2}(50 \mathrm{ml})$ was added dropwise over 30 minutes under a nitrogen atmosphere. The stirring was continued for additional $30 \mathrm{~min}$. To the oil, obtained after evaporation of the reaction mixture, ethyl acetate $(10 \mathrm{ml})$ followed by hexane $(30 \mathrm{ml})$ were added. The precipitated solid was filtered off and purified through flash column or preparative thin layer chromatography.

6,10-diphenyl-2,3,6a,7,12,13-hexahydro-7,13-ethenoimidazo [1',2':1,2][1,3,5]triazino[3,4$d][1,2,4]$ triazolino[1,2-a][1,2,4]triazine- $6(5 H), 9(8 H), 11(10 H)$-trione $\quad(7 a) . \quad$ Two chromatographic purifications were needed, the first one with ethyl acetate/hexane $3: 1 \rightarrow 4: 1 \mathrm{v} / \mathrm{v}$ yielded $360 \mathrm{mg}$ of roughly purified product, second one was performed with diethyl ether/dichloromethane/ethyl acetate 4:1:1 v/v/v. White solid, yield 23\%, $280 \mathrm{mg}, \mathrm{mp}$ (dec.): 198-199 ${ }^{\circ} \mathrm{C}$; IR $\left(v_{\max }, \mathrm{cm}^{-1}\right)$ 3094, 3061, 2960, 2895, 1777 (C=O urazole), 1712 (C=O urazole), $1688(\mathrm{C} 5=\mathrm{O}), 1665(\mathrm{C}=\mathrm{N}), 1397 .{ }^{1} \mathrm{H}$ NMR $\left(200 \mathrm{MHz}, \mathrm{DMSO}-d_{6}\right): \delta_{\mathrm{H}} 3.62-3.90\left(4 \mathrm{H}, \mathrm{m},-\mathrm{CH}_{2}-\right.$ $\mathrm{CH}_{2}$ - imidaz.), 4.53-4.57 (1H, m, H7), $5.96(1 \mathrm{H}, \mathrm{d}, J=2.5 \mathrm{~Hz}, \mathrm{H6a}), 6.40(1 \mathrm{H}, \mathrm{dd}, J=5.2,1.5$ $\mathrm{Hz}, \mathrm{H} 13), 6.43-6.50$ (1H, m, H16), 7.16 (1H, ddd, $J=7.8,5.2,1.5 \mathrm{~Hz}, \mathrm{H} 15), 7.34-7.57(10 \mathrm{H}$, m, $2 \times \mathrm{Ph}$ ). ${ }^{13} \mathrm{C}$ NMR (50 MHz, DMSO- $d_{6}$ ): $\delta_{\mathrm{C}} 44.7$ (C3), 50.8 (C2), 52.3 (C7), 62.0 (C13), 67.2 (C6a), 125.3 (C16), 126.2, 128.5, 128.9, 129.1, 129.7 (C aromat), 133.1 (C15), 137.4 (C aromat), $149.0(\mathrm{C}=\mathrm{N}), 151.2(\mathrm{C} 5=\mathrm{O}), 154.0,155.5(2 \times \mathrm{C}=\mathrm{O}$ urazole $)$; Anal. Calcd for $\mathrm{C}_{23} \mathrm{H}_{19} \mathrm{~N}_{7} \mathrm{O}_{3}$ (441.44): C, 62.58; H, 4.34; N, 22.21\%. Found: C, 62.42, H, 4.32; N, 22.18\%

6-(4-methylphenyl)-10-phenyl-2,3,6a,7,12,13-hexahydro-7,13-ethenoimidazo [1',2':1,2] $[1,3,5]$ triazino[3,4-d][1,2,4]triazolino[1,2-a][1,2,4]triazine-6(5H),9(8H),11(10H)-trione $(7 \mathrm{~b})$. Eluent: ethyl acetate/hexane 3:2 $\rightarrow 4: 1 \mathrm{v} / \mathrm{v}$. White solid, yield 24\%, $290 \mathrm{mg}, \mathrm{mp}(\mathrm{dec}$.): 199-200 ${ }^{\circ} \mathrm{C}$, IR $\left(v_{\max }, \mathrm{cm}^{-1}\right): 3072,2987,2881,1781(\mathrm{C}=\mathrm{O}$ urazole), $1721(\mathrm{C}=\mathrm{O}$ urazole $), 1702(\mathrm{C} 5=\mathrm{O})$, $1659(\mathrm{C}=\mathrm{N}), 1398,775,727 .{ }^{1} \mathrm{H}$ NMR $\left(500 \mathrm{MHz}, \mathrm{DMSO}-d_{6}\right): \delta_{\mathrm{H}} 2.37\left(3 \mathrm{H}, \mathrm{s}, \mathrm{CH}_{3}\right), 3.62-3.67$ $(1 \mathrm{H}, \mathrm{m}, \mathrm{H} 3), 3.77(2 \mathrm{H}, \mathrm{t}, J=8.3 \mathrm{~Hz}, 2 \times \mathrm{H} 2), 3.84-3.79(1 \mathrm{H}, \mathrm{m}, \mathrm{H} 3$ '), $4.55(1 \mathrm{H}, \mathrm{dd}, J=3.4,2.0$ $\mathrm{Hz}, \mathrm{H} 7), 5.92(1 \mathrm{H}, \mathrm{d}, J=2.4 \mathrm{~Hz}, \mathrm{H6a}), 6.37(1 \mathrm{H}, \mathrm{d}, J=4.9 \mathrm{~Hz}, \mathrm{H} 13), 6.47(1 \mathrm{H}, \mathrm{t}, J=6.8 \mathrm{~Hz}$, H16), 7.14-7.16 (1H, m, H15), 7.22-7.23 (2H, m, Ph), $7.32\left(2 \mathrm{H}, \mathrm{d}, J=7.6 \mathrm{~Hz}, \mathrm{C}_{6} \mathrm{H}_{4}\right), 7.39(2 \mathrm{H}$, d, $\left.J=7.6 \mathrm{~Hz}, \mathrm{C}_{6} \mathrm{H}_{4}\right), 7.43-7.46(1 \mathrm{H}, \mathrm{m}, \mathrm{Ph}), 7.49-7.52(2 \mathrm{H}, \mathrm{m}, \mathrm{Ph})$. 
${ }^{13} \mathrm{C}$ NMR $\left(50 \mathrm{MHz}, \mathrm{DMSO}-d_{6}\right): \delta_{\mathrm{C}} 20.7\left(\mathrm{CH}_{3}\right), 44.7(\mathrm{C} 3), 50.8(\mathrm{C} 2), 52.3(\mathrm{C} 7), 62.0(\mathrm{C} 13), 67.2$ (C6a), 125.4 (C16), 126.2, 128.4, 128.9, 129.2, 130.2, 130.6 (10 C aromat), 133.0 (C15), 134.8, 138.1 (2C aromat), $149.1(\mathrm{C}=\mathrm{N}), 151.3(\mathrm{C} 5=\mathrm{O}), 154.1,155.3(2 \times \mathrm{C}=\mathrm{O}$ urazole $)$. Anal. Calcd for $\mathrm{C}_{24} \mathrm{H}_{21} \mathrm{~N}_{7} \mathrm{O}_{3}$ (455.47): C, 63.29; H, 4.65; N, 21.53\%; Found: C, 63.23; H, 4.59; N, 21.48\%.

6-(3-methylphenyl)-10-phenyl-2,3,6a,7,12,13-hexahydro-7,13-ethenoimidazo[1',2':1,2] $[1,3,5]$ triazino $[3,4-d][1,2,4]$ triazolino $[1,2-a][1,2,4]$ triazine-6(5H),9(8H),11(10H)-trione $(7 \mathrm{c})$. Eluents: ethyl acetate/hexane 6:1 v/v until the first stereoisomer was collected followed by ethyl acetate/methanol 9:0.5 v/v which enabled elution of the second stereoisomer. The evaporated solids were washed with acetone/diethyl ether $1: 1 \mathrm{v} / \mathrm{v}(10 \mathrm{ml})$, filtered off and dried.

Stereoisomer 1: white solid, yield 42\%, $490 \mathrm{mg}, \mathrm{mp}$ (dec.): $218-220{ }^{\circ} \mathrm{C}$; IR $\left(v_{\max }, \mathrm{cm}^{-1}\right) 3058$, 2987, 2950, 2877, 1787 (C=O urazole), 1727 ( $\mathrm{C}=\mathrm{O}$ urazole), 1689 (C5=O), $1654(\mathrm{C}=\mathrm{N}), 1401$, 721. ${ }^{1} \mathrm{H}$ NMR $\left(200 \mathrm{MHz}, \mathrm{DMSO}-d_{6}\right): \delta_{\mathrm{H}} 2.36\left(3 \mathrm{H}, \mathrm{s}, \mathrm{CH}_{3}\right), 3.61-3.89\left(4 \mathrm{H}, \mathrm{m},-\mathrm{CH}_{2}-\mathrm{CH}_{2}-\right.$ imidaz.), 4.53-4.58 (1H, m, H7), $5.92(1 \mathrm{H}, \mathrm{d}, J=2.2 \mathrm{~Hz}, \mathrm{H} 6 \mathrm{a}), 6.39$ (1H, dd, $J=5.1,1.5 \mathrm{~Hz}$, H13), 6.45-5.52 (1H, m, H16), 7.12-7.19 (3H, m, H15+2H aromat.), 7.27 (1H, d, J = 7.6 Hz, H aromat. $\left.\mathrm{C}_{6} \mathrm{H}_{4} \mathrm{Me}\right), 7.37-7.54\left(6 \mathrm{H}, \mathrm{m}\right.$, aromat.). ${ }^{13} \mathrm{C} \mathrm{NMR}\left(50 \mathrm{MHz}, \mathrm{DMSO}-d_{6}\right): \delta_{\mathrm{C}} 20.8\left(\mathrm{CH}_{3}\right)$, 44.7 (C3), 50.8 (C2), 52.3 (C7), 62.0 (C13), 67.2 (C6a), 125.4 (C16), 126.2, 128.9, 129.0, 129.2, $129.3,129.5,130.6$ (10 C aromat), 133.0 (C15), 137.3, 139.4 (2C aromat.), $149.0(\mathrm{C}=\mathrm{N}), 151.3$ $(\mathrm{C} 5=\mathrm{O}), 154.1,155.3\left(2 \times \mathrm{C}=\mathrm{O}\right.$ urazole). Anal. Calcd for $\mathrm{C}_{24} \mathrm{H}_{21} \mathrm{~N}_{7} \mathrm{O}_{3}(455,47) \mathrm{C}, 63.29 ; \mathrm{H}, 4.65$; N, 21.53; Found: C, 63.32; H, 4.61; N, 21.56\%.

Stereoisomer 2: white solid, yield $8 \%, 90 \mathrm{mg}, \mathrm{mp}$ (dec.): 190-192 ${ }^{\circ} \mathrm{C}$; IR $\left(v_{\max }, \mathrm{cm}^{-1}\right) 3075$, 3001, 2923, 2874, 1778 (C=O urazole), 1729 (C=O urazole), $1712(\mathrm{C} 5=\mathrm{O}), 1689(\mathrm{C}=\mathrm{N}), 1400$, 774. ${ }^{1} \mathrm{H}$ NMR $\left(200 \mathrm{MHz}, \mathrm{DMSO}-d_{6}\right): \delta_{\mathrm{H}} 2.33\left(3 \mathrm{H}, \mathrm{s}, \mathrm{CH}_{3}\right), 3.65-3.88\left(4 \mathrm{H}, \mathrm{m},-\mathrm{CH}_{2}-\mathrm{CH}_{2}-\right.$ imidaz.) $4.58(1 \mathrm{H}, \mathrm{d}, J=5.9 \mathrm{~Hz}, \mathrm{H} 7), 5.32(1 \mathrm{H}, \mathrm{s}, \mathrm{H} 6 \mathrm{a}), 6.50(1 \mathrm{H}, \mathrm{dd}, J=4.5, \sim 1 \mathrm{~Hz}, \mathrm{H} 13)$, 6.56-6.67 (1H, m, H16), 6.91-7.01 (1H, m, H15), 7.19-7.51 (9H, m, Ph + H aromat. $\left.\mathrm{C}_{6} \mathrm{H}_{4} \mathrm{Me}\right)$; ${ }^{13} \mathrm{C}$ NMR (50 MHz, DMSO- $\left.d_{6}\right): \delta_{\mathrm{C}} 20.85\left(\mathrm{CH}_{3}\right), 45.2(\mathrm{C} 3), 50.7(\mathrm{C} 2), 52.8(\mathrm{C} 7), 62.4(\mathrm{C} 13)$, 67.0 (C6a), 126.2, 127.4 (C aromat.), 128.7 (C16), 129.1 (C aromat.), 129.7 (C15), 130.7, 137.1, 138.9 (C aromat.), $149.2(\mathrm{C}=\mathrm{N}), 152.1(\mathrm{C} 5=\mathrm{O}), 154.2,156.1(2 \times \mathrm{C}=\mathrm{O}$, urazole $)$. Anal. Calcd for $\mathrm{C}_{24} \mathrm{H}_{21} \mathrm{~N}_{7} \mathrm{O}_{3}(455,47) \mathrm{C}, 63.29 ; \mathrm{H}, 4.65 ; \mathrm{N}, 21.53$; Found: C, 63.20; H, 4.66; N, $21.52 \%$.

6-(3,4-dimethylphenyl)-10-phenyl-2,3,6a,7,12,13-hexahydro-7,13-ethenoimidazo[1',2':1,2] $[1,3,5]$ triazino $[3,4-d][1,2,4]$ triazolino[1,2-a][1,2,4]triazine-6(5H),9(8H),11(10H)-trione $(7 \mathrm{~d})$. Eluents: ethyl acetate/hexane $5: 1(\mathrm{v} / \mathrm{v}) \rightarrow$ ethyl acetate, two fractions were collected. The evaporated solids are washed with acetone/diethyl ether $1: 1 \mathrm{v} / \mathrm{v}(10 \mathrm{ml})$, filtered off and dried.

Stereoisomer 1: white solid, yield 58\%, $700 \mathrm{mg}, \mathrm{mp}$ (dec.): $225-226{ }^{\circ} \mathrm{C}$; IR $\left(v_{\max }, \mathrm{cm}^{-1}\right): 3057$, 2927, 2879, 1788 (C=O urazole), $1728\left(\mathrm{C}=\mathrm{O}\right.$ urazole), $1687(\mathrm{C} 5=\mathrm{O}), 1655(\mathrm{C}=\mathrm{N}), 1401 .{ }^{1} \mathrm{H}$ NMR (500 MHz, DMSO- $\left.d_{6}\right): \delta_{\mathrm{H}} 2.27\left(3 \mathrm{H}, \mathrm{s}, \mathrm{CH}_{3}\right), 2.28\left(3 \mathrm{H}, \mathrm{s}, \mathrm{CH}_{3}\right), 3.62-3.67(1 \mathrm{H}, \mathrm{m}, \mathrm{H} 3)$, 3.75-3.79 (2H, m, 2×H2), 3.83-3.89 (1H, m, H3'), 4.55-4.56 (1H, m, H7), 5.89 (1H, d, J = 2.4 Hz, H6a), 6.38 (1H, dd, $J=4.9, ~ 1 ~ H z, H 13), 6.47-6.50(1 \mathrm{H}, \mathrm{m}, \mathrm{H} 16), 7.02-7.12(2 \mathrm{H}, \mathrm{m}, \mathrm{H}$ aromat. $\left.\mathrm{C}_{6} \mathrm{H}_{3} \mathrm{Me}_{2}\right), 7.14-7.17(1 \mathrm{H}, \mathrm{m}, \mathrm{H} 15), 7.27\left(1 \mathrm{H}, \mathrm{d}, J=8.3 \mathrm{~Hz}, \mathrm{H}\right.$ aromat. $\left.\mathrm{C}_{6} \mathrm{H}_{3} \mathrm{Me}_{2}\right), 7.37$ $(1 \mathrm{H}, \mathrm{d}, J=7.3 \mathrm{~Hz}, \mathrm{Ph}), 7.40\left(1 \mathrm{H}, \mathrm{s}, \mathrm{H}\right.$ aromat. $\left.\mathrm{C}_{6} \mathrm{H}_{3} \mathrm{Me}_{2}\right), 7.45(1 \mathrm{H}, \mathrm{t}, J=7.3 \mathrm{~Hz}, \mathrm{Ph}), 7.51(1 \mathrm{H}$, $\mathrm{t}, J=7.3 \mathrm{~Hz}, \mathrm{Ph}) .{ }^{13} \mathrm{C}$ NMR spectrum could not be measured because of the poor solubility of 
the compound in DMSO. Anal. Calcd for $\mathrm{C}_{25} \mathrm{H}_{23} \mathrm{~N}_{7} \mathrm{O}_{3}$ (469.50): C, 63.96; H, 4.94; N, 20.88\%. Found: C, 63.88; H, 4.90; N, 20.93\%.

Stereoisomer 2: white solid, yield $8 \%, 90 \mathrm{mg}$; $\mathrm{mp}$ (dec.): $211-212{ }^{\circ} \mathrm{C}$; IR $\left(v_{\max }, \mathrm{cm}^{-1}\right): 3072$, 2993, 2874, $1777\left(\mathrm{C}=\mathrm{O}\right.$ urazole), $1712(\mathrm{C}=\mathrm{O}), 1655(\mathrm{C}=\mathrm{N}), 1397 .{ }^{1} \mathrm{H}$ NMR $(500 \mathrm{MHz}$, DMSO$\left.d_{6}\right): \delta_{\mathrm{H}} 2.23\left(3 \mathrm{H}, \mathrm{s}, \mathrm{CH}_{3}\right), 2.26\left(3 \mathrm{H}, \mathrm{s}, \mathrm{CH}_{3}\right), 3.67-3.72(1 \mathrm{H}, \mathrm{m}, \mathrm{H} 3), 3.74-3.84(2 \mathrm{H}, \mathrm{m}, 2 \times \mathrm{H} 2)$, $3.88-3.93(1 \mathrm{H}, \mathrm{m}, \mathrm{H3}$ '), $4.58(1 \mathrm{H}, \mathrm{d}, J=6.3 \mathrm{~Hz}, \mathrm{H} 7), 5.28(1 \mathrm{H}, \mathrm{d}, J=1.5 \mathrm{~Hz}, \mathrm{H6a}), 6.48(1 \mathrm{H}$, $\mathrm{dd}, J=5.4,1.5 \mathrm{~Hz}, \mathrm{H} 13), 6.61(1 \mathrm{H}, \mathrm{dt}, J=7.1,1.5 \mathrm{~Hz}, \mathrm{H} 16), 6.95(1 \mathrm{H}, \mathrm{dt}, J=6.6,1.5 \mathrm{~Hz}$, H15), $7.12\left(1 \mathrm{H}, \mathrm{d}, J=8.1 \mathrm{~Hz}, \mathrm{H}\right.$ aromat. $\left.\mathrm{C}_{6} \mathrm{H}_{3} \mathrm{Me}_{2}\right), 7.21\left(1 \mathrm{H}, \mathrm{s}, \mathrm{H}\right.$ aromat. $\left.\mathrm{C}_{6} \mathrm{H}_{3} \mathrm{Me}_{2}\right), 7.23(1 \mathrm{H}$, d, $J=8.1 \mathrm{~Hz}, \mathrm{H}$ aromat. $\left.\mathrm{C}_{6} \mathrm{H}_{3} \mathrm{Me}_{2}\right), 7.37(2 \mathrm{H}, \mathrm{d}, J=7.3 \mathrm{~Hz}, \mathrm{Ph}), 7.43(1 \mathrm{H}, \mathrm{t}, \mathrm{J}=7.3 \mathrm{~Hz}, \mathrm{Ph})$, $7.49(1 \mathrm{H}, \mathrm{t}, J=7.3 \mathrm{~Hz}, \mathrm{Ph}) .{ }^{13} \mathrm{C}$ NMR $\left(50 \mathrm{MHz}, \mathrm{DMSO}-d_{6}\right): \delta_{\mathrm{H}} 19.0\left(\mathrm{CH}_{3}\right), 19.4\left(\mathrm{CH}_{3}\right), 45.2$ (C3), 50.6 (C2), 52.9 (C7), 62.4 (C13), 67.1 (C6a), 126.2, 126.5, 127.4 (4C aromat.), 128.7 (C16), 129.1 (2C aromat.), 129.7 (C15), 129.9, 130.2, 130.7, 134.7, 136.6, 137.4 (6C aromat.), $149.2(\mathrm{C}=\mathrm{N}), 152.2(\mathrm{C} 5=\mathrm{O}), 154.2,156.1(2 \times \mathrm{C}=\mathrm{O}$, urazole $)$. Anal. Calcd for $\mathrm{C}_{25} \mathrm{H}_{23} \mathrm{~N}_{7} \mathrm{O}_{3}$ (469.50): C, 63.96; H, 4.94; N, 20.88\%. Found: C, 63.98; H, 4.97; N, $20.84 \%$.

6-(4-chlorophenyl)-10-phenyl-2,3,6a,7,12,13-hexahydro-7,13-ethenoimidazo[1',2':1,2] $[1,3,5]$ triazino $[3,4-d][1,2,4]$ triazolino[1,2-a][1,2,4]triazine-6(5H),9(8H),11(10H)-trione $(7 \mathrm{e})$. Eluents: ethyl acetate/hexane 8:3 v/v until the first stereoisomer was collected followed by ethyl acetate/methanol 9:0.5 v/v which enabled elution of the second stereoisomer.

Stereoisomer 1: white solid, yield 32\%, $390 \mathrm{mg}, \mathrm{mp}$ (dec.) 194-196 ${ }^{\circ} \mathrm{C}$; IR $\left(v_{\max }, \mathrm{cm}^{-1}\right): 3055$, 2979, 2911, 1779 (C=O urazole), $1717(\mathrm{C}=\mathrm{O}$ urazole $), 1700(\mathrm{C}=\mathrm{O}), 1690(\mathrm{C}=\mathrm{O}), 1667(\mathrm{C}=\mathrm{N})$. ${ }^{1} \mathrm{H}$ NMR $\left(500 \mathrm{MHz}, \mathrm{DMSO}-d_{6}\right): \delta_{\mathrm{H}} 3.62-3.67(1 \mathrm{H}, \mathrm{m}, \mathrm{H} 3), 3.76-3.79(2 \mathrm{H}, \mathrm{m}, 2 \times \mathrm{H} 2), 3.85-3.90$ $(1 \mathrm{H}, \mathrm{m}, \mathrm{H} 3$ '), 4.66-4.67 (1H, m, H7), $5.97(1 \mathrm{H}, \mathrm{d}, J=2.4 \mathrm{~Hz}, \mathrm{H} 6 \mathrm{a}), 6.4(1 \mathrm{H}, \mathrm{d}, J=5.0 \mathrm{~Hz}$, H13), $6.47(1 \mathrm{H}, \mathrm{t}, J=6.8 \mathrm{~Hz}, \mathrm{H} 16), 7.15(1 \mathrm{H}, \mathrm{ddd}, J=6.8,5,1.5 \mathrm{~Hz}, \mathrm{H} 15), 7.38-7.41(4 \mathrm{H}, \mathrm{m}$, aromat.), $7.45(1 \mathrm{H}, \mathrm{t}, J=7.3 \mathrm{~Hz}, \mathrm{Ph}), 7.5(2 \mathrm{H}, \mathrm{t}, J=7.3 \mathrm{~Hz}, \mathrm{Ph}), 7.60(1 \mathrm{H}, \mathrm{d}, J=8.8 \mathrm{~Hz}$, $\left.\mathrm{C}_{6} \mathrm{H}_{4} \mathrm{Cl}\right) ;{ }^{13} \mathrm{C}$ NMR (125 MHz, DMSO- $\left.d_{6}\right): \delta_{\mathrm{C}} 44.9$ (C3), $51.0(\mathrm{C} 2), 52.4(\mathrm{C} 7), 62.2(\mathrm{C} 13), 67.2$ (C6a), 125.5 (C16), 126.4, 129.1, 129.4, 130.0, 130.7 (11C, aromat), 133.3 (C15), 136.5 (1C aromat), $149.1(\mathrm{C}=\mathrm{N}), 151.3(\mathrm{C} 5=\mathrm{O}), 154.3,155.4(2 \times \mathrm{C}=\mathrm{O}$, urazole $) ; \mathrm{MS}, \mathrm{m} / \mathrm{z}(\%)=248$ $\left(\mathrm{C}_{11} \mathrm{H}_{9} \mathrm{ClN} \mathrm{N}_{4} \mathrm{O}, 56\right), 250\left(\mathrm{C}_{11} \mathrm{H}_{9} \mathrm{ClN}_{4} \mathrm{O}+2,18\right), 227\left(\mathrm{C}_{12} \mathrm{H}_{9} \mathrm{~N}_{3} \mathrm{O}_{2}, 100\right), 138$ (75), 119 (65). Anal. Calcd for $\mathrm{C}_{23} \mathrm{H}_{18} \mathrm{ClN}_{7} \mathrm{O}_{3}$ (475.89) C, 58.05; H, 3.81; N, 20.60; Found: C, 58.12; H, 3.76; N, $20.59 \%$.

Stereoisomer 2: white solid, yield $8 \%, 96 \mathrm{mg}, \mathrm{mp}(\mathrm{dec}) 211-.213{ }^{\circ} \mathrm{C}$; IR $\left(v_{\max }, \mathrm{cm}^{-1}\right): 3037$, 2993, 2874, $1775(\mathrm{C}=\mathrm{O}$ urazole $), 1736(\mathrm{C}=\mathrm{O}$ urazole $), 1711(\mathrm{C} 5=\mathrm{O}), 1659(\mathrm{C}=\mathrm{N}), 1395 .{ }^{1} \mathrm{H}$ NMR (500 MHz, DMSO- $\left.d_{6}\right): \delta_{\mathrm{H}} 3.68-3.73(1 \mathrm{H}, \mathrm{m}, \mathrm{H} 3), 3.75-3.85(2 \mathrm{H}, \mathrm{m}, 2 \times \mathrm{H} 2), 3.90-3.97$ $(1 \mathrm{H}, \mathrm{m}, \mathrm{H} 3$ ') $, 4.65(1 \mathrm{H}, \mathrm{d}, J=5.9 \mathrm{~Hz}, \mathrm{H} 7), 5.38(1 \mathrm{H}, \mathrm{s}, \mathrm{H6a}), 6.49(1 \mathrm{H}, \mathrm{d}, J=5.4 \mathrm{~Hz}, \mathrm{H} 13)$, 6.59-6.62 (1H, m, H16), 6.95-6.98 (1H, m, H15), $7.35\left(2 \mathrm{H}, \mathrm{d}, J=7.8 \mathrm{~Hz}, \mathrm{C}_{6} \mathrm{H}_{4} \mathrm{Cl}\right), 7.42-7.51$ $(5 \mathrm{H}, \mathrm{m}, \mathrm{Ph}), 7.55\left(2 \mathrm{H}, \mathrm{d}, J=7.8 \mathrm{~Hz}, \mathrm{C}_{6} \mathrm{H}_{4} \mathrm{Cl}\right) .{ }^{13} \mathrm{C}$ NMR $\left(125 \mathrm{MHz}, \mathrm{DMSO}-d_{6}\right) \delta 45.2(\mathrm{C} 3)$, 50.7 (C2), 52.6 (C7), 62.5 (C13), 66.9 (C6a), 126.3, 127.4 (3C aromat.), 128.8 (C16), 129.2, 129.4 (4C aromat.), 129.9 (C15), 130.7, 131.3, 133.0, 136.2 (5C aromat.), $149.1(\mathrm{C}=\mathrm{N}), 152.0$ $(\mathrm{C} 5=\mathrm{O}), 154.2,156.1(2 \times \mathrm{C}=\mathrm{O}$, urazole $) . \mathrm{MS}, \mathrm{m} / z(\%)=248\left(\mathrm{C}_{11} \mathrm{H}_{9} \mathrm{ClN}_{4} \mathrm{O}, 73\right), 250$ 
$\left(\mathrm{C}_{11} \mathrm{H}_{9} \mathrm{ClN}_{4} \mathrm{O}+2,24\right), 227\left(\mathrm{C}_{12} \mathrm{H}_{9} \mathrm{~N}_{3} \mathrm{O}_{2}, 77\right), 138(100), 119$ (63). Anal. Calcd for $\mathrm{C}_{23} \mathrm{H}_{18} \mathrm{ClN}_{7} \mathrm{O}_{3}$ (475.89) C, 58.05; H, 3.81; N, 20.60; Found: C, 57.89; H, 3.78; N, $20.62 \%$.

\section{6-(3-chlorophenyl)-10-phenyl-2,3,6a,7,12,13-hexahydro-7,13-ethenoimidazo[1',2':1,2]} $[1,3,5]$ triazino $[3,4-d][1,2,4]$ triazolino $[1,2-a][1,2,4]$ triazine-6(5H),9(8H),11(10H)-trione $(7 f)$. Eluent: ethyl acetate/hexane 3:1 v/v. White solid, yield 24\%, $330 \mathrm{mg}, \mathrm{mp}$ (dec.) $191-193{ }^{\circ} \mathrm{C}$; IR $\left(v_{\max }, \mathrm{cm}^{-1}\right): 3088,3037,2920,2871,1776(\mathrm{C}=\mathrm{O}$ urazole $), 1721(\mathrm{C}=\mathrm{O}$ urazole $), 1693(\mathrm{C} 5=\mathrm{O})$, $1662(\mathrm{C}=\mathrm{N}), 1453,1398,773,745 .{ }^{1} \mathrm{H}$ NMR $\left(500 \mathrm{MHz}, \mathrm{DMSO}-d_{6}\right): \delta_{\mathrm{H}} 3.62-3.68(1 \mathrm{H}, \mathrm{m}, \mathrm{H} 3)$, $3.78(2 \mathrm{H}, \mathrm{t}, J=8.3 \mathrm{~Hz}, 2 \times \mathrm{H} 2), 3.85-3.90\left(1 \mathrm{H}, \mathrm{m}, \mathrm{H} 3{ }^{\prime}\right), 4.68(1 \mathrm{H}, \mathrm{dt}, J=5.4,1.5 \mathrm{~Hz}, \mathrm{H} 7), 5.98$ $(1 \mathrm{H}, \mathrm{d}, J=2.4 \mathrm{~Hz}, \mathrm{H6a}), 6.40(1 \mathrm{H}, \mathrm{dd}, J=4.9,1.5 \mathrm{~Hz} \mathrm{H} 13), 6.47-6.49(1 \mathrm{H}, \mathrm{m}, \mathrm{H} 16), 7.15(1 \mathrm{H}$, ddd, $J=7.8,4.9,1.5 \mathrm{~Hz}, \mathrm{H} 15), 7.31-7.36\left(1 \mathrm{H}, \mathrm{m}, \mathrm{C}_{6} \mathrm{H}_{4} \mathrm{Cl}\right), 7.4\left(1 \mathrm{H}, \mathrm{d}, J=8.3 \mathrm{~Hz}, \mathrm{C}_{6} \mathrm{H}_{4} \mathrm{Cl}\right)$, $7.41\left(1 \mathrm{H}, \mathrm{s}, \mathrm{C}_{6} \mathrm{H}_{4} \mathrm{Cl}\right), 7.44-7.47(1 \mathrm{H}, \mathrm{m}, \mathrm{Ph}), 7.50-7.56(4 \mathrm{H}, \mathrm{m}, \mathrm{Ph}) .{ }^{13} \mathrm{C}$ NMR $(50 \mathrm{MHz}$, DMSO- $d_{6}$ ): $\delta_{\mathrm{C}} 44.7$ (C3), 50.8 (C2), 52.2 (C7), 62.0 (C13), 67.0 (C6a), 125.3 (C16), 126.2, 127.5, 128.6, 128.8, 128.9, 129.2, 130.6, 131.2 (10 C aromat.), 133.1 (C15), 133.7, 138.8 (2C aromat.), $148.8(\mathrm{C}=\mathrm{N}), 151.1(\mathrm{C} 5=\mathrm{O}), 154.1,155.3(2 \times \mathrm{C}=\mathrm{O}$, urazole $)$. Anal. Calcd for $\mathrm{C}_{23} \mathrm{H}_{18} \mathrm{ClN}_{7} \mathrm{O}_{3}$ (475.89): C, 58.05; H, 3.81; N, 20.60\%. Found: C, 58.01; H, 3.77; N, 20.64\%.

6-(3,4-dichlorophenyl)-10-phenyl-2,3,6a,7,12,13-hexahydro-7,13-ethenoimidazo[1',2':1,2] $[1,3,5]$ triazino[3,4-d][1,2,4]triazolino[1,2-a][1,2,4]triazine-6(5H),9(8H),11(10H)-trione $(7 \mathrm{~g})$. Eluents: ethyl acetate/hexane $3: 2 \rightarrow 4: 1 \mathrm{v} / \mathrm{v}$. Two fractions were collected. The evaporated solids were washed with ethyl acetate, filtered off and dried.

Stereoisomer 1: white solid, yield $34 \%, 440 \mathrm{mg}, \mathrm{mp}(\mathrm{dec}):. 214-215^{\circ} \mathrm{C}$; IR $\left(v_{\max }, \mathrm{cm}^{-1}\right): 3058$, 3026, 2928, 2878, 1786 ( $\mathrm{C}=\mathrm{O}$ urazole), $1728(\mathrm{C}=\mathrm{O}$ urazole $), 1691(\mathrm{C} 5=\mathrm{O}), 1656(\mathrm{C}=\mathrm{N}), 1401$. ${ }^{1} \mathrm{H}$ NMR $\left(500 \mathrm{MHz}\right.$, DMSO- $\left.d_{6}\right): \delta_{\mathrm{H}} 3.62-3.67(1 \mathrm{H}, \mathrm{m}, \mathrm{H} 3), 3.73-3.82(1 \mathrm{H}, \mathrm{m}, 2 \times \mathrm{H} 2), 3.85-3.90$ $\left(1 \mathrm{H}, \mathrm{m}, \mathrm{H} 3{ }^{\prime}\right), 4.84-4.86(1 \mathrm{H}, \mathrm{m}, \mathrm{H} 7), 5.97(1 \mathrm{H}, \mathrm{d}, J=2 \mathrm{~Hz}, \mathrm{H6a}), 6.39(1 \mathrm{H}, \mathrm{d}, J=4.9 \mathrm{~Hz}$, H13), 6.46-6.48 (1H, m, H16), 7.12-7.15 (1H, m, H15), $7.36\left(1 \mathrm{H}, \mathrm{dd}, J=8.3, \sim 1 \mathrm{~Hz}, \mathrm{C}_{6} \mathrm{H}_{3} \mathrm{Cl}_{2}\right)$, $7.40(2 \mathrm{H}, \mathrm{d}, J=7.8 \mathrm{~Hz}, \mathrm{Ph}), 7.45(1 \mathrm{H}, \mathrm{t}, J=7.8 \mathrm{~Hz}, \mathrm{Ph}), 7.52(2 \mathrm{H}, \mathrm{t}, J=7.8 \mathrm{~Hz}, \mathrm{Ph}), 7.74(1 \mathrm{H}$, d, $\left.J \sim 1 \mathrm{~Hz}, \mathrm{C}_{6} \mathrm{H}_{3} \mathrm{Cl}_{2}\right), 7.80\left(1 \mathrm{H}, \mathrm{d}, J=8.3 \mathrm{~Hz}, \mathrm{C}_{6} \mathrm{H}_{3} \mathrm{Cl}_{2}\right) .{ }^{13} \mathrm{C} \mathrm{NMR}\left(50 \mathrm{MHz}\right.$, DMSO- $\left.d_{6}\right): \delta_{\mathrm{C}} 44.6$ (C3), 50.8 (C2), 52.0 (C7), 62.0 (C13), 66.9 (C6a), 125.4 (C16), 126.2, 128.9, 129.0, 129.2, 130.6, 130.7, 131.4, 131.5, 131.9 (11C, aromat.), 133.1 (C15), 137.3 (1C aromat.), $148.7(\mathrm{C}=\mathrm{N})$, $151.0(\mathrm{C} 5=\mathrm{O}), 154.1,155.2\left(2 \times \mathrm{C}=\mathrm{O}\right.$, urazole). Anal. Calcd for $\mathrm{C}_{23} \mathrm{H}_{17} \mathrm{Cl}_{2} \mathrm{~N}_{7} \mathrm{O}_{3}$ (510.33): $\mathrm{C}$, $54.13 ; \mathrm{H}, 3.36$; N, $19.21 \%$. Found: C, 54.06; H, 3.29; N, $19.05 \%$.

Strereoisomer 2: white solid, yield 6\%, $80 \mathrm{mg}, \mathrm{mp}$ (dec.): 200-201; IR $\left(v_{\max }, \mathrm{cm}^{-1}\right): 3071,2988$, 2876, $1782(\mathrm{C}=\mathrm{O}$ urazole $), 1721(\mathrm{C}=\mathrm{O}$ urazole $), 1704(\mathrm{C} 5=\mathrm{O}), 1664(\mathrm{C}=\mathrm{N}), 1399 .{ }^{1} \mathrm{H}$ NMR (500 MHz, DMSO- $\left.d_{6}\right): \delta_{\mathrm{H}} 3.67-3.73(1 \mathrm{H}, \mathrm{m}, \mathrm{H} 3), 3.75-3.85(2 \mathrm{H}, \mathrm{m}, 2 \times \mathrm{H} 2), 3.90-3.95(1 \mathrm{H}, \mathrm{m}$, H3'), $4.75(1 \mathrm{H}, \mathrm{d}, J=6.3 \mathrm{~Hz}, \mathrm{H} 7), 5.37(1 \mathrm{H}, \mathrm{s}, \mathrm{H} 6 \mathrm{a}), 6.50(1 \mathrm{H}, \mathrm{d}, J=4.9 \mathrm{~Hz}, \mathrm{H} 13), 6.58-6.60$ $(1 \mathrm{H}, \mathrm{m}, \mathrm{H} 16), 6.97(1 \mathrm{H}, \mathrm{t}, J=6.3 \mathrm{~Hz}, \mathrm{H} 15), 7.35-7.37(2 \mathrm{H}, \mathrm{m}, J=7.8 \mathrm{~Hz}, \mathrm{H}$ aromat.), $7.42-$ 7.45 (2H, m, H aromat.), 7.48-7.51 (2H, m, H aromat.), $7.76\left(1 \mathrm{H}, \mathrm{d}, J=8.3 \mathrm{~Hz}, \mathrm{C}_{6} \mathrm{H}_{3} \mathrm{Cl}_{2}\right), 7.82$ $\left(1 \mathrm{H}, \mathrm{d}, J \sim 1 \mathrm{~Hz}, \mathrm{C}_{6} \mathrm{H}_{3} \mathrm{Cl}_{2}\right) ;{ }^{13} \mathrm{C}$ NMR (50 MHz, DMSO- $\left.d_{6}\right): \delta_{\mathrm{C}} 45.1(\mathrm{C} 3), 50.7(\mathrm{C} 2), 52.5(\mathrm{C} 7)$, 62.4 (C13), 67.1 (C6a), 126.2, 127.3 (3C aromat.), 128.7 (C16), 128.9, 129.1 (3C aromat.), 129.9 (C15), 130.2, 130.7, 131.0, 131.3, 131.6, 137.2 (6C aromat.), $148.9(\mathrm{C}=\mathrm{N}), 151.9(\mathrm{C} 5=\mathrm{O}), 154.1$, 
156.0 ( $2 \times \mathrm{C}=\mathrm{O}$, urazole). Anal. Calcd for $\mathrm{C}_{23} \mathrm{H}_{17} \mathrm{Cl}_{2} \mathrm{~N}_{7} \mathrm{O}_{3}$ (510.33): C, 54.13; H, 3.36; N, $19.21 \%$. Found: C, 54.02; H, 3.32; N, 19.23\%.

\section{6-(4-methoxyphenyl)-10-phenyl-2,3,6a,7,12,13-hexahydro-7,13-ethenoimidazo[1',2':1,2]}

$[1,3,5]$ triazino $[3,4-d][1,2,4]$ triazolino[1,2-a][1,2,4]triazine-6(5H),9(8H),11(10H)-trione $(7 \mathrm{~h})$. Eluents: ethyl acetate/hexane $4: 1 \mathrm{v} / \mathrm{v} \rightarrow$ ethyl acetate. The evaporated solid was washed with acetone/diethyl ether 1:1 v/v (10 ml), filtered off and dried. White solid, yield 46\%, $560 \mathrm{mg}, \mathrm{mp}$ (dec.): 202-203 ${ }^{\circ} \mathrm{C}$; IR $\left(v_{\max }, \mathrm{cm}^{-1}\right): 3119,2936,2889,1781$ (C=O urazole), 1720 ( $\mathrm{C}=\mathrm{O}$ urazole), $1697(\mathrm{C} 5=\mathrm{O}), 1659(\mathrm{C}=\mathrm{N}), 1401,1243 .{ }^{1} \mathrm{H}$ NMR $\left(500 \mathrm{MHz}, \mathrm{DMSO}-d_{6}\right): \delta_{\mathrm{H}} 3.61-3.67(1 \mathrm{H}, \mathrm{m}$, H3), 3.57-3.78 (2H, m, 2×H2), $3.81\left(3 \mathrm{H}, \mathrm{s}, \mathrm{CH}_{3}\right), 3.81-3.89(1 \mathrm{H}, \mathrm{m}, \mathrm{H} 3$ ') , 4.56-4.57 $(1 \mathrm{H}, \mathrm{m}$, H7), $5.88(1 \mathrm{H}, \mathrm{d}, J=2 \mathrm{~Hz}, \mathrm{H6a}), 6.39(1 \mathrm{H}, \mathrm{dd}, J=4.9, \sim 1 \mathrm{~Hz}, \mathrm{H} 13), 6.48(1 \mathrm{H}, \mathrm{t}, J=6.8 \mathrm{~Hz}$, $\mathrm{H} 16), 7.06\left(2 \mathrm{H}, \mathrm{d}, J=8.8 \mathrm{~Hz}, \mathrm{H}\right.$ aromat. $\left.\mathrm{C}_{6} \mathrm{H}_{4} \mathrm{OMe}\right), 7.14-7.17(1 \mathrm{H}, \mathrm{m}, \mathrm{H} 15), 7.24-7.30(2 \mathrm{H}$, m, $\mathrm{H}$ aromat. $\left.\mathrm{C}_{6} \mathrm{H}_{4} \mathrm{OMe}\right), 7.40(2 \mathrm{H}, \mathrm{d}, J=7.3 \mathrm{~Hz}, \mathrm{Ph}), 7.45(1 \mathrm{H}, \mathrm{t}, J=7.3 \mathrm{~Hz}, \mathrm{Ph}), 7.51(2 \mathrm{H}, \mathrm{t}, J$ $=7.3 \mathrm{~Hz}, \mathrm{Ph}) .{ }^{13} \mathrm{C}$ NMR $\left(50 \mathrm{MHz}, \mathrm{DMSO}-d_{6}\right): \delta_{\mathrm{C}} 44.7(\mathrm{C} 3), 50.8(\mathrm{C} 2), 52.4(\mathrm{C} 7), 55.4\left(\mathrm{CH}_{3}\right)$, 62.0 (C13), 67.4 (C6a), 114.9 (2C aromat.), 125.4 (C16), 126.2, 128.9, 129.2, 129.7, 129.8, 130.6 (9C aromat.), $133.0(\mathrm{C} 15), 149.1(\mathrm{C}=\mathrm{N}), 151.5(\mathrm{C} 5=\mathrm{O}), 154.1,155.3(2 \times \mathrm{C}=\mathrm{O}$, urazole $)$, 159.0 (1C aromat.). Anal. Calcd for $\mathrm{C}_{24} \mathrm{H}_{21} \mathrm{~N}_{7} \mathrm{O}_{4}$ (471.47): $\mathrm{C}, 61.14 ; \mathrm{H}, 4.49 ; \mathrm{N}, 20.80 \%$. Found: C, 61.18; H, 4.32; N, 20.69\%.

\section{General procedure of preparing the reduced cycloadducts $(8,9 a)$ and $(9 b)$}

The procedure is based on the one reported in ref. 22 . To the cycloadduct $\mathbf{6}, 7 \mathbf{b}$ or $7 \mathbf{e}(0.3 \mathrm{mmol})$ dissolved in $\mathrm{CH}_{2} \mathrm{Cl}_{2}(\sim 1.5 \mathrm{ml}) o$-nitrobenzenesulfonylhydrazide ${ }^{28}(0.33 \mathrm{~g}, 1.5 \mathrm{mmol})$ was added followed by triethylamine $(0.24 \mathrm{~g}, 0.33 \mathrm{ml}, 2.4 \mathrm{mmol})$. The resulting slurry was stirred for 20 hours at room temperature. The homogenous reaction mixture was then diluted with $\mathrm{CH}_{2} \mathrm{Cl}_{2}(10$ $\mathrm{ml})$ and washed successively with saturated sodium bicarbonate solution $(7 \mathrm{ml})$ and water $(7 \mathrm{ml})$. The organic phase was dried over $\mathrm{MgSO}_{4}$, concentrated under reduced pressure and purified by flash chromatography.

\section{2-phenyl-2,3,6,7,8a,9,14,15-octahydro-9,15-ethanodiimidazo[1',2':1,2;1",2”:5,6]} $[1,3,5]$ triazino[3,4-d][1,2,4]triazolino[1,2-a][1,2,4]triazine-11(10H),13(12H)-dion (8). Eluent: dichloromethane/acetone/methanol 3:2:0.8. White solid, yield 50\%, $45 \mathrm{mg}, \mathrm{mp}$ (dec.): 230-232 ${ }^{\circ} \mathrm{C}$; IR $\left(v_{\max }, \mathrm{cm}^{-1}\right): 2994,2945,2880,1776(\mathrm{C}=\mathrm{O}$ urazole), 1716 (C=O urazole), 1670 (C5=O), $1646(\mathrm{C}=\mathrm{N}), 1410,773,692 .{ }^{1} \mathrm{H}$ NMR $\left(200 \mathrm{MHz}, \mathrm{DMSO}-d_{6}\right): \delta_{\mathrm{H}} 2.05-2.28(\mathrm{~m}, 4 \mathrm{H}$, $2 \times \mathrm{H} 17+2 \times \mathrm{H} 18), 2.77-2.95(\mathrm{~m}, 1 \mathrm{H}, \mathrm{H} 7), 3.25-3.80(\mathrm{~m}, 7 \mathrm{H}, 2 \times \mathrm{H} 2,2 \times \mathrm{H} 3,2 \times \mathrm{H} 6, \mathrm{H} 7$ '), 4.62-4.46 (m, 1H, H8a/H9), 4.74 (br. s, 1H, H8a/H9), 5.86 (br. s, $1 \mathrm{H}, \mathrm{H} 15), 7.38-7.60$ (m, 5H, Ph). ${ }^{13} \mathrm{C}$ NMR (50 MHz, DMSO- $\left.d_{6}\right): \delta_{\mathrm{c}} 16.5$ (C17/C18), 24.5 (C17/C18), 45.9 (C3), 48.6 (C7), 50.5 (C6), 51.0 (C2), 51.2 (C9), 61.3 (C15), 68.7 (C8a), 126.6, 128.6, 129.0, 131.1 (6C, Ph), 148.5 $(\mathrm{C} 16 \mathrm{a}=\mathrm{N}), 153.5(\mathrm{C} 4 \mathrm{a}=\mathrm{N}), 154.3,156.2(2 \times \mathrm{C}=\mathrm{O}$ urazole $)$. Anal. Calcd for $\mathrm{C}_{19} \mathrm{H}_{20} \mathrm{~N}_{8} \mathrm{O}_{2}(392.41)$ : C, 58.15; H, 5.14; N, 28.55\%. Found: C, 58.01; H, 5.22; N, 28.87\%.

6-(4-methylphenyl)-10-phenyl-2,3,6a,7,12,13-hexahydro-7,13-ethanoimidazo[1',2':1,2] $[1,3,5]$ triazino $[3,4-d][1,2,4]$ triazolino[1,2-a][1,2,4]triazine-6(5H),9(8H),11(10H)-trione $(9 a)$. Eluent: ethyl acetate/hexane 4:1 v/v. White solid, yield 93\%, $130 \mathrm{mg}, \mathrm{mp}(\mathrm{dec}$.$) 201-203 { }^{\circ} \mathrm{C}$; IR 
$\left(v_{\max }, \mathrm{cm}^{-1}\right): 3034,2920,2862,1718(\mathrm{C}=\mathrm{O}$ urazole $), 1723(\mathrm{C}=\mathrm{O}$ urazole $), 1702$ (C5=O), 1664 $(\mathrm{C}=\mathrm{N}), 1408,767 .{ }^{1} \mathrm{H}$ NMR $\left(200 \mathrm{MHz}, \mathrm{DMSO}-d_{6}\right): \delta_{\mathrm{H}} 1.95-2.34(4 \mathrm{H}, \mathrm{m}, 2 \times \mathrm{H} 12+2 \times \mathrm{H} 13), 2.34$ $\left(3 \mathrm{H}, \mathrm{s}, \mathrm{CH}_{3}\right), 3.60-3.93\left(5 \mathrm{H}, \mathrm{m},-\mathrm{CH}_{2}-\mathrm{CH}_{2}\right.$ - imidaz. $\left.+\mathrm{H} 7\right), 5.82(1 \mathrm{H}, \mathrm{s}, \mathrm{H} 13), 5.96(1 \mathrm{H}, \mathrm{s}, \mathrm{H} 6 \mathrm{a})$, 7.22-7.27 (4H, m, aromat.), 7.43-7.52 (5H, m, aromat.). ${ }^{13} \mathrm{C}$ NMR (50 MHz, DMSO- $d_{6}$ ): $\delta_{\mathrm{c}} 16.0$ (C15/16), $20.6\left(\mathrm{CH}_{3}\right), 24.4$ (C15/16), 44.7 (C3), 50.1 (C2), 50.7 (C7), 61.1 (C13), 67.6 (C6a), 126.6, 127.8, 128.6, 128.9, 130.0, 131.0, 134.8, $137.6\left(12 \mathrm{C}, \mathrm{Ph}+\mathrm{C}_{6} \mathrm{H}_{4}\right), 148.1(\mathrm{C} 14 \mathrm{a}=\mathrm{N}), 152.0$ (C5=O), 153.4, $153.8\left(2 \times \mathrm{C}=\mathrm{O}\right.$ urazole). Anal. Calcd for $\mathrm{C}_{24} \mathrm{H}_{23} \mathrm{~N}_{7} \mathrm{O}_{3}$ (457.48): C, 63.01; H, 5.07; N, 21.43\%. Found: C, 63.11; H, 4.88; N, 21.41\%.

\section{6-(4-chlorophenyl)-10-phenyl-2,3,6a,7,12,13-hexahydro-7,13-ethanoimidazo[1',2':1,2]} $[1,3,5]$ triazino[3,4-d][1,2,4] triazolino[1,2-a][1,2,4]triazine-6(5H),9(8H),11(10H)-trione $(9 \mathrm{~b})$. Eluents: ethyl acetate/hexane $6: 1 \rightarrow$ ethyl acetate.

Stereoisomer 1: Eluents: ethyl acetate/hexane 6:1 $\rightarrow$ ethyl acetate. White solid, yield 55\%, 145 $\mathrm{mg}, \mathrm{mp}$ (dec.): $235-337{ }^{\circ} \mathrm{C}$ (acetonitrile); IR $\left(v_{\max }, \mathrm{cm}^{-1}\right): 3072,2951,2878,1778(\mathrm{C}=\mathrm{O}$ urazole $), 1718(\mathrm{C}=\mathrm{O}$, urazole $), 1649(\mathrm{C} 5=\mathrm{O}), 1656(\mathrm{C}=\mathrm{N}), 1492,1416,1397,763 .{ }^{1} \mathrm{H}$ NMR (500 MHz, DMSO- $\left.d_{6}\right): \delta_{\mathrm{H}} 1.94-1.99(1 \mathrm{H}, \mathrm{m}), 2.07-2.12(1 \mathrm{H}, \mathrm{m}), 2.18-2.23(1 \mathrm{H}, \mathrm{m}), 2.32-2.37(1 \mathrm{H}$, m) $(2 \times \mathrm{H} 15+2 \times \mathrm{H} 16), 3.61-3.66(1 \mathrm{H}, \mathrm{m}, \mathrm{H} 3), 3.69-3.78(2 \mathrm{H}, \mathrm{m}, 2 \times \mathrm{H} 2), 3.85-3.90(1 \mathrm{H}, \mathrm{m}, \mathrm{H} 3$ '), $4.01(1 \mathrm{H}, \mathrm{s}, \mathrm{H} 7), 5.87(1 \mathrm{H}, \mathrm{s}, \mathrm{H} 13), 5.96(1 \mathrm{H}, \mathrm{s}, \mathrm{H6a}), 7.42\left(2 \mathrm{H}, \mathrm{d}, J=8.8 \mathrm{~Hz}, \mathrm{C}_{6} \mathrm{H}_{4} \mathrm{Cl}\right), 7.45-$ $7.48(1 \mathrm{H}, \mathrm{m}, \mathrm{Ph}), 7.51-7.56(4 \mathrm{H}, \mathrm{m}, \mathrm{Ph}), 7.58\left(2 \mathrm{H}, \mathrm{d}, J=8.8 \mathrm{~Hz}, \mathrm{C}_{6} \mathrm{H}_{4} \mathrm{Cl}\right) .{ }^{13} \mathrm{C}$ NMR $(50 \mathrm{MHz}$, DMSO- $\left.d_{6}\right): \delta_{\mathrm{c}} 16.0(\mathrm{C} 15 / 16), 24.4$ (C15/16), 44.7 (C3), 50.0 (C2), 50.7 (C7), $61.2(\mathrm{C} 13), 67.3$ (C6a), 126.5, 128.6, 129.0, 129.5, 129.9, 131.0, 132.6, $136.4\left(12 \mathrm{C}, \mathrm{Ph}+\mathrm{C}_{6} \mathrm{H}_{4} \mathrm{Cl}\right), 147.9$ $(\mathrm{C} 14 \mathrm{a}=\mathrm{N}), 151.7(\mathrm{C} 5=\mathrm{O}), 153.5,153.9\left(2 \times \mathrm{C}=\mathrm{O}\right.$ urazole). Anal. Calcd for $\mathrm{C}_{23} \mathrm{H}_{20} \mathrm{ClN}_{7} \mathrm{O}_{3}$ (477.90): C, 57.80; H, 4.22; N, 20.52\%. Found: C, 57.63; H, 4.20; N, 20.74\%.

Stereoisomer 2: Eluents: ethyl acetate/hexane 6:1 $\rightarrow$ ethyl acetate. Yellow solid, yield 45\%, 65 mg, mp (dec.): $214-215^{\circ} \mathrm{C}$; IR ( $\left.v_{\max }, \mathrm{cm}^{-1}\right): 3061,2953,2879,1781$ (C=O urazole), $1713(\mathrm{C}=\mathrm{O}$ urazole $+\mathrm{C} 5=\mathrm{O}), 1664(\mathrm{C}=\mathrm{N}), 1493,1406,768 .{ }^{1} \mathrm{H}$ NMR $\left(500 \mathrm{MHz}, \mathrm{DMSO}-d_{6}\right): \delta_{\mathrm{H}} 1.99-2.26$ (4H, m, $2 \times \mathrm{H} 15+2 \times \mathrm{H} 16), 3.65-3.75\left(3 \mathrm{H}, \mathrm{m},-\mathrm{CH}_{2}-\mathrm{CH}_{2}\right.$ - imidaz.), 3.86-3.91 (1H, m, $-\mathrm{CH}_{2}-\mathrm{CH}_{2}-$ imidaz.), 4.19 (1H, br. s, H7), $5.74(1 \mathrm{H}, \mathrm{s}, \mathrm{H6a}), 6.11(1 \mathrm{H}, \mathrm{br} . \mathrm{s}, \mathrm{H} 13), 7.39(2 \mathrm{H}, \mathrm{d}, J=8.3 \mathrm{~Hz}$, $\left.\mathrm{C}_{6} \mathrm{H}_{4} \mathrm{Cl}\right), 7.42-7.54\left(9 \mathrm{H}, \mathrm{m}, \mathrm{Ph}+\mathrm{C}_{6} \mathrm{H}_{4} \mathrm{Cl}\right) .{ }^{13} \mathrm{C}$ NMR (50 MHz, DMSO- $\left.d_{6}\right): \delta_{\mathrm{c}} 21.2(\mathrm{C} 15 / 16), 25.2$ (C15/16), 44.9 (C3), 49.4 (C2), 50.5 (C7), 62.3 (C13), 70.0 (C6a), 126.3, 128.3, 129.0, 130.7, 131.3, 132.4, $136.3\left(12 \mathrm{C}, \mathrm{Ph}+\mathrm{C}_{6} \mathrm{H}_{4} \mathrm{Cl}\right), 149.1(\mathrm{C} 14 \mathrm{a}=\mathrm{N}), 151.7,152.0(3 \mathrm{C}, \mathrm{C} 5=\mathrm{O}, 2 \times \mathrm{C}=\mathrm{O}$ urazole). Anal. Calcd for $\mathrm{C}_{23} \mathrm{H}_{20} \mathrm{ClN}_{7} \mathrm{O}_{3}$ (477.90): C, 57.80; H, 4.22; N, 20.52\%. Found: $\mathrm{C}$, $57.63 ; \mathrm{H}, 4.16 ; \mathrm{N}, 20.44 \%$.

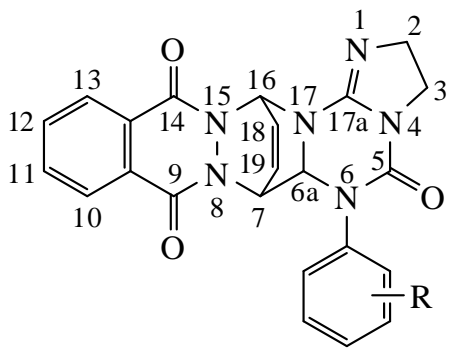

Figure 5. Numbering of atoms used to describe ${ }^{1} \mathrm{H}$ and ${ }^{13} \mathrm{C}$ NMR spectra of compounds 11a-b. 


\section{General procedure of preparing compounds (11a-b)}

To a cooled $\left(-20^{\circ} \mathrm{C}\right)$ and stirred solution of the appropriate 6-aryl-2,3-dihydro-6a $H$-imidazo[1,2a]pyrido[1,2-c][1,3,5]triazin-5(6H)-one $(1.8 \mathrm{mmol})$ in $\mathrm{CH}_{2} \mathrm{Cl}_{2}(30 \mathrm{ml})$ a cold solution of phthalazine-1,4-dione 5 in acetonitrile (prepared from phthalhydrazide ( $2.9 \mathrm{~g}, 18 \mathrm{mmol})$ and lead tetraacetate $(7.94 \mathrm{~g}, 18 \mathrm{mmol})$ according to Clement ${ }^{24}$ ) was added dropwise until no further consumption of the dienophile was observed. The reaction mixture was filtered under reduced pressure and the solution evaporated to give a solid residue that was subjected to preparative thin layer chromatography.

\section{6-(4-methylphenyl)-2,3,6a,7,16,17-hexahydro-7,16-ethenoimidazo[1',2':1,2]}

\section{$[1,3,5]$ triazino[3,4-d]phthalazino[2,3-a][1,2,4]triazine-5(6H),9(8H),14(15H)-trione}

(11a).

The first chromatographic separation was performed with ethyl acetate/methanol 9:1. The last fraction collected was purified in the next chromatographic separation using dichloromethane/methanol 5:2 v/v as the eluent to afford the desired cycloadduct.

Yellow solid, yield 15\%, $120 \mathrm{mg}, \mathrm{mp} 220-222{ }^{\circ} \mathrm{C}$; IR $\left(v_{\max }, \mathrm{cm}^{-1}\right): 3050,2923,2872,1698$ $(\mathrm{C}=\mathrm{O}), 1681(\mathrm{C}=\mathrm{O}), 1654(\mathrm{C}=\mathrm{N}), 1396,1380,755,699 .{ }^{1} \mathrm{H}$ NMR (500 MHz, DMSO- $\left.d_{6}\right): \delta_{\mathrm{H}}$ $2.40\left(3 \mathrm{H}, \mathrm{s}, \mathrm{CH}_{3}\right), 3.60-3.64(1 \mathrm{H}, \mathrm{m}, \mathrm{H} 3), 3.74-3.81(2 \mathrm{H}, \mathrm{m}, 2 \times \mathrm{H} 2), 3.82-3.88$ (1H, m, H3'), 5.53-5.55 (1H, m, H7), $5.91(1 \mathrm{H}, \mathrm{d}, J=2 \mathrm{~Hz}, \mathrm{H6a}), 6.63(1 \mathrm{H}, \mathrm{t}, J=6.8 \mathrm{~Hz}, \mathrm{H} 19), 7.22-7.24$ (3H, m, H16+2H aromat.), $7.30(1 \mathrm{H}, \mathrm{t}, J=6.8 \mathrm{~Hz}, \mathrm{H} 18), 7.35(2 \mathrm{H}, \mathrm{d}, J=8.3 \mathrm{~Hz}$, aromat.), 7.93-7.95 (2H, m, aromat.), 8.12-8.14 (1H, m, aromat.), 8.20-8.22 (1H, m, aromat). ${ }^{13} \mathrm{C} \mathrm{NMR}$ (50 MHz, DMSO- $\left.d_{6}\right): \delta_{\mathrm{C}} 20.7\left(\mathrm{CH}_{3}\right), 44.7$ (C3), 48.7 (C2), 50.8 (C7), 58.4 (C16), 68.0 (C6a), 127.2, 127.3, 128.0, 128.1, 130.2, 134.0, 134.1, 134.9, 138.0 (14C, aromat.+C18+C19), 149.0 $(\mathrm{C}=\mathrm{N}), 151.2(\mathrm{C} 5=\mathrm{O}), 152.9,153.6(2 \times \mathrm{C}=\mathrm{O}$ of 2,3-dihydrophthalazine-1,4-dione $) . \mathrm{MS}, \mathrm{m} / z(\%)$ $=228\left(\mathrm{C}_{12} \mathrm{H}_{12} \mathrm{~N}_{4} \mathrm{O}, 88\right), 212\left(\mathrm{C}_{12} \mathrm{H}_{8} \mathrm{~N}_{2} \mathrm{O}_{2}, 70\right), 118$ (100), 104 (56). Anal. Calcd for $\mathrm{C}_{24} \mathrm{H}_{20} \mathrm{~N}_{6} \mathrm{O}_{3}$ (440.45): C, 65.45; H, 4.58; N, 19.08\%. Found: C, 65.37; H, 4.53; N, $19.00 \%$.

\section{6-(3,4-dichlorophenyl)-2,3,6a,7,16,17-hexahydro-7,16-ethenoimidazo[1',2':1,2]}

\section{$[1,3,5]$ triazino[3,4- $d]$ phthalazino[2,3-a][1,2,4]triazine-5(6H),9(8H),14(15H)-trione}

(11b).

The first chromatographic separation was performed with ethyl dichloromethane/ethyl acetate $1: 1$. The last fraction collected was purified in the next chromatographic separation using dichloromethane/methanol 10:1 v/v as the eluent to afford the desired cycloadduct.

Yellow solid, yield 20\%, $180 \mathrm{mg}$, mp (dec.) 182-184; IR $\left(v_{\max }, \mathrm{cm}^{-1}\right): 3070,3037,2881,1706$ $(\mathrm{C}=\mathrm{O}), 1683(\mathrm{C} 5=\mathrm{O}), 1660(\mathrm{C}=\mathrm{N}), 1473,1451,1320,1132,699 .{ }^{1} \mathrm{H}$ NMR $(200 \mathrm{MHz}, \mathrm{DMSO}-$ $\left.d_{6}\right): \delta_{\mathrm{H}} 3.66-3.89\left(4 \mathrm{H}, \mathrm{m},-\mathrm{CH}_{2}-\mathrm{CH}_{2^{-}}\right.$imidaz.), 5.64-5.67 (1H, m, H7), 5.98 (d, J = 2.2 Hz, H6a), 6.63 (dt, 1H, $J=6.1,1.5 \mathrm{~Hz}, \mathrm{H} 19), 7.20-7.31$ (m, 2H, H16+1H aromat.), 7.36-7.41 (m, $1 \mathrm{H}, \mathrm{H} 18$ ), 7.75 (d, 1H, $J=2.2 \mathrm{~Hz}$, aromat.), 7.83 (dd, $1 \mathrm{H}, J=8.5, \sim 1 \mathrm{~Hz}$, aromat.), 7.92-7.97 (m, 2H, aromat.), 8.13-8.23 (m, 2H, aromat.). ${ }^{13} \mathrm{C}$ NMR (50 MHz, DMSO- $\left.d_{6}\right): \delta_{\mathrm{C}} 44.7$ (C3), 48.8 (C2), 50.8 (C7), 58.4 (C10), 67.7 (C6a), 127.1, 127.28, 127.33, 127.97, 128.0, 128.9, 130.5, 313.3, 131.5, 131.9, 134.1, 134.2, 135.0, $137.5(14 \mathrm{C}$, aromat. $+\mathrm{CH}=\mathrm{CH}), 148.6(\mathrm{C}=\mathrm{N}), 150.8$ $(\mathrm{C} 5=\mathrm{O}), \quad 152.9,153.7 \quad(2 \times \mathrm{C}=\mathrm{O}$ of 2,3-dihydrophthalazine-1,4-dione $)$. Anal. Calcd for $\mathrm{C}_{23} \mathrm{H}_{16} \mathrm{Cl}_{2} \mathrm{~N}_{6} \mathrm{O}_{3}$ (495.32): C, 55.77; H, 3.26; N, 16.97\%. Found: C, 55.61; H, 3.22; N, $16.92 \%$. 
X-ray crystal structure analysis. The diffraction data for single crystals of compound $\mathbf{6}$ were collected at $130 \mathrm{~K}$ with Oxford Diffraction XCaliburE diffractometer using Mo K $\alpha$ radiation and those of compound $\mathbf{9 b}$ at $293 \mathrm{~K}$ with an Oxford Diffraction SuperNova diffractometer using $\mathrm{Cu}$ $\mathrm{K} \alpha$ radiation. The intensity data were collected and processed using CrysAlisPro Software. The structures were solved by direct methods with the program SHELXS-97 ${ }^{29}$ and refined by fullmatrix least-squares method on $F^{2}$ with SHELXL-97. ${ }^{29}$ Crystallographic data for compounds 6 and 9b have been deposited in the Cambridge Crystallographic Data Centre, with the deposition Nos CCDC 814026 \& 814027.

Crystal data for 6: $\mathrm{C}_{19} \mathrm{H}_{18} \mathrm{~N}_{8} \mathrm{O}_{2} \cdot \mathrm{C}_{6} \mathrm{H}_{6} \cdot \mathrm{H}_{2} \mathrm{O}$, monoclinic, space group $C 2 / c, a=28.1800(9), b=$ 9.5506(3), $c=19.5038(7) \AA, \beta=117.485(4)^{\circ}, V=4656.7(3) \AA^{3}, Z=8, T=130 \mathrm{~K}, d_{x}=1.388 \mathrm{~g}$ $\mathrm{cm}^{-3}, \mu(\mathrm{Mo} \mathrm{K} \alpha)=0.096 \mathrm{~mm}^{-1}, 13385$ data were collected up to $\theta_{\max }=26.37^{\circ}\left(R_{\text {int }}=0.0191, R_{\sigma}\right.$ $=0.0285)$. Final $R$ indices for 3660 reflections with $I>2 \sigma(I)$ and 334 refined parameters are: $R_{I}$ $=0.0388, w R_{2}=0.1047\left(R_{1}=0.0511, w R_{2}=0.1082\right.$ for all 4741 data $)$. Water molecule is disordered over two sites with equal occupancies.

Crystal data for 9b: $\mathrm{C}_{23} \mathrm{H}_{20} \mathrm{ClN}_{7} \mathrm{O}_{3}$, orthorhombic, space group Pbca, $a=14.5913(2), b=$ 7.9820(1), $c=36.2167(4) \AA, V=4218.08(9) \AA^{3}, Z=8, T=293 \mathrm{~K}, d_{x}=1.505 \mathrm{~g} \mathrm{~cm}^{-3}, \mu(\mathrm{Cu}$ $\mathrm{K} \alpha)=1.981 \mathrm{~mm}^{-1}, 61573$ data were collected up to $\theta_{\max }=73.83^{\circ}\left(R_{\text {int }}=0.0321, R_{\sigma}=0.0096\right)$. Final $R$ indices for 4071 reflections with $I>2 \sigma(I)$ and 308 refined parameters are: $R_{1}=0.0344$, $w R_{2}=0.0957\left(R_{1}=0.0353, w R_{2}=0.0965\right.$ for all 4237 data $)$.

Cytotoxic activity. All cell lines were obtained from the German Collection of Microorganisms and Cell Cultures (DSMZ) (Braunschweig, Germany). Cytotoxicity studies were performed with a well-established microtiter assay based on the staining of adherent cells with crystal violet; the method has been described in detail in previous publications. ${ }^{25}$ DMSO stock solutions of the compounds were diluted 1000-fold in cell culture medium (RPMI 1640 medium supplemented with $10 \%$ fetal calf serum) to give the final test concentration. Five, 2-fold dilutions of test substance where used in each experiment (i.e., 20, 10, 5.0, 2.5, $1.25 \mu \mathrm{M}$ ). Untreated controls received only DMSO (0.1\%). Cells were continuously exposed to compounds for $96 \mathrm{~h}$ at $37^{\circ} \mathrm{C}$ in a humid atmosphere of $5 \% \mathrm{CO}_{2} /$ air. The $\mathrm{IC}_{50}$ values were estimated by least squares analysis of the dose-response curves to give the concentration of substance that inhibits cell growth by $50 \%$ compared to untreated controls. Reported $\mathrm{IC}_{50}$ values are the averages of 3-6 independent determinations.

\section{Acknowledgements}

AM thanks the Erasmus Program of the EU for supporting her stay in Greifswald. 


\section{References}

1. (a) Toyoda, T.; Brobey, R. K. B.; Sano, G.-I.; Horii, T.; Tomioka, N.; Itai, A. Biochem. Biophys. Res. Comm. 1997, 235, 515. (b) Itai, A., Toyoda, T. Japanese Patent 10310 526, 1998; Chem. Abstr. 1999, 130, 62956. (c) Gulyás, G.; Emri, T.; Simon, A.; Györgydeák, Z. Folia Microbiol. (Praha) 2002, 47, 29. (d) Dolzhenko, A. V.; Chui, W.-K.; Dolzhenko, A. V.; Chan, L.-W. J. Fluor. Chem. 2005, 126, 795. (e) Dolzhenko, A. V.; Chui, W.-K. J. Heterocycl. Chem. 2006, 43, 95. (f) Dolzhenko, A. V., Chui, W.-K., Dolzhenko, A. V. J. Heterocycl. Chem. 2006, 43, 1513.

2. Novellino, E.; Abignete, E.; Cosimelli, B.; Greco, G.; Iadanza, M.; Laneri, S.; Lavecchia, A.; Rimoli, M. G. J. Med. Chem. 2002, 45, 5030.

3. (a) Kim, S.-H.; Bartholomew, D. G.; Allen L. B. J. Med. Chem. 1978, 21, 883. (b) Golankiewicz, B.; Januszczyk, P.; Ikeda, S.; Balzarini, J.; De Clerq, E. J. Med. Chem. 1995, 38, 3558. (c). Ojwang, J. O; Ali, S.; Smee, D. F.; Morrey, J. D.; Shimasaki, C. D.; Sidwell, R. W. Antiviral Res. 2005, 68, 49. (d) Dukhan, D.; Leroy, F.; Peyronnet, J.; Bosc, E.; Chaves, D.; Durka, M.; Storer, R.; La Colla, P.; Gosselin, G. Nuclosides, Nuclotides and Nucleic Acids 2005, 24, 671.

4. Luengo, J. I.; Duffy, K. J. US Patent 6346531 B1, 2002.

5. (a) Heider, J.; Austel, V.; Hanel, N.; Noll, K. et al., Ger. Pat. 3443 812, 1986; Chem. Abstr. 1986, 105, 153064k. (b) Sączewski, F.; Nasal, A. Acta Pol. Pharm. - Drug Research 1993, 50, 337. (c) Sączewski, F.; Nasal, A. Acta Pol. Pharm. - Drug Research 1995, 52, 237.

6. Heider, J.; Austel, V.; Hanel, N.; Noll, K. et al. Ger. Pat. 346 778, 1986; Chem. Abstr. 1986, 105, 172459j.

7. Matosiuk, D. M.; Fidecka, S.; Antkiewicz-Michaluk, L.; Lipkowski, J.; Dybała, I.; Kozioł, A. E. Eur. J. Med. Chem. 2002, 37, 761.

8. (a) Bekircan, O.; Küxük, M.; Kahveci, B.; Kolayli S. Arch. Pharm. Chem. Life Sci. 2005, 338, 365. (b) Łakomska, I.; Golankiewicz, B.; Wietrzyk, J.; Pełczyńska, M.; Nasulewicz, A.; Opolski, A.; Sitkowski, J.; Kozerski, L.; Szłyk, E. Inorg. Chim. Acta. 2005, 358, 1911. c) Sączewski, F.; Maruszak, M.; Bednarski, P. J. Arch. Pharm. Chem. Life Sci. 2008, 341, 121.

9. Sączewski, F.; Foks, H. Synthesis 1981, 154.

10. Sączewski, F.; Gdaniec, M.; Ośmiałowski, K. J. Chem. Soc, Perkin Trans. 1 1987, 1033.

11. (a) Schenker, K.; Druey, J. Helv. Chim. Acta 1959, 42, 1971. (b) Buchi, G.; Coffen, D. L.; Kocsis, K.; Sonnet, P. E.; Ziegler, F. E. J. Am. Chem. Soc. 1965, 87, 2073. (c) Weinstein, B.; Chang Lin, L.-Ch.; Fowler, F. W. J. Org. Chem. 1980, 45, 1657. (d) Sundberg, R. J.; Bloom, J. D. J. Org. Chem. 1980, 45, 3382. (e) Sundberg, R. J.; Bloom, J. D. J. Org. Chem. 1981, 46, 4863. (e) Buchi, G.; Coffen, D. L.; Kocsis, K.; Sonnet, P. E.; Ziegler, F. E. J. Am. Chem. Soc. 1996, 88, 2532. (g) Nakano, H.; Tsugawa, N.; Takahashi, K.; Okuyama, Y.; Fujita, R. Tetrahedron 2006, 62, 10879. (h) Nakano, H.; Tsugawa, N.; Fujita, R. Tetrahedron Lett. 2005, 46, 5677. 
12. (a) Tomisawa, H.; Hongo, H., Tetrahedron Lett. 1969, 29, 2465. (b) Mariano, P. S.; Huesmann, P. L.; Beamer, R. L.; Dunaway-Mariano, D. Tetrahedron, 1978, 34, 2617. (c) Tomisawa, H.; Nakano, H.; Hongo, H. Heterocycles 1990, 30, 359. (d) Posner, G. H.; Vinader, V.; Afarinkia, K. J. Org. Chem. 1992, 57, 4088. (e) Afarinkia, K.; Mahmood, F. Tetrahedron Lett. 1998, 39, 493.

13. Tomisawa, H.; Nakano, H.; Hongo, H. Chem. Pharm. Bull. 1988, 36, 1692.

14. iboga alkaloids: (a) Popik, P.; Layer, R. T.; Skolnick, P. Pharmcol. Rev. 1995, 47, 235. (b) Popik, P.; Glick, S. D. Drugs Future 1996, 21, 1109. (c) Popik, P. Life Sciences 1996, 59, PL-379. (d) Popik, P.; Layer, R. T.; Skolnick, P. in The Alkaloids, Cordell, G.A. Ed.; Academic Press: San Diego, 1998; Vol. 52, pp 197-231. (e) Levi, M. S.; Borne, R. F. Curr. Med. Chem. 2002, 9, 1807. (f) He, D.-Y.; McGough, N. N. H.; Ravindranathan, A.; Jeanblanc, J.; Logrip, M. L.; Phamloung, K.; Janak, P. H.; Ron, D. J. Neurosc. 2005, 25, 619. (g) Sundberg, R. J.; Smith, S. Q. in The Alkaloids, Cordell, G.A. Ed.; Elsevier, 2002, Vol. 59, pp 281-376. dioscorine: (h) Broadbent, J. L.; Schnieden, H. Br. J. Pharamcol. 1958, 13, 213. (h) Nagata, K.; Aistrup, G. L.; Honda, H.; Shono, T.; Narahashi, T. Pestic. Biochem. Physiol. 1999, 64, 157.

15. Iwu, M. M.U. S. Patent 5019 580, 1991; Chem. Abstr. 1992, 116, 76378.

16. (a) Khan, M.O. F.; Levi, M. S.; Clark, C. R.; Ablordeppey, S. Y.; Law, S.-J.; Wilson, N. H.; Borne, R. F. in Studies in Natural Products Chemistry, ur-Rahman, A. Ed.; Elsevier, 2008, Vol. 34, pp 753-787. (b) Iriepa, I.; Villasante, F. J.; Gálvez, E.; Labeaga, L.; Innerarity, A.; Orjales, A. Bioorg. Med. Chem. Lett. 2002, 12, 189. (c) Tomiyama, T.; Tomiyama, A.; Imamaki, T.; Ueyama, N.; Sonegawa, M.; Takeuchi, S. U. S. Pat. 6124 460, 2000; Chem. Abstr. 2000, 133, 17677p. (d) Yonan, P. K. U. S. Pat. 4134 890, 1979. (e) Scherico Ltd., U. S. Pat. 1095 105, 1965; Chem. Abstr. 1967, 66, 37787a. (f) Plumpe, H.; Puls, W. U. S. Pat. 3764 605, 1973. (g) Tomiyama, H.; Kobayashi, Y.; Noda, A.U. S. Pat. 6903111 B2, 2005. (h) Villani, F. J. Canadian Pat. CA 828 738, 1969; Chem. Abstr. 1969, 71, 124281c. (i) Bernardi, L.; Bertazzoli, C.; Chieli, T.; Maggioni, P.U. S. Pat. 3 709 893, 1973. (j) Yokota, M.; Takizawa, E.; Ohkura, Y.; Fukai, C.; Tomiyama, T. Eur. J. Med. Chem. 1997, 32, 377. (k) Adelstein, G. W.; Yen, Ch. H.; Dajani, E. Z.; Bianchi, R. G. J. Med. Chem. 1976, 19, 1221. (1) Adelstein, G. W. U. S. Pat. 4028 364, 1977; Chem. Abstr. 1977, 87, 84848q. (m) Pfizer inc. Eur. Pat. Appl. 0356193 A2, 1989. (n) Khan, M. O. F.; Levi, M. S.; Tekwani, B. L.; Wilson, N. H., Borne, R. F. Bioorg. Med. Chem. 2007, 15, 3919.

17. (a) Matsumara, Y.; Nakamura, Y.; Maki, T; Onomura, O. Tetrahedron Lett. 2000, 41, 7685.

(b) Takenaka, N.; Huang, Y.; Rawal, V. H. Tetrahedron Lett. 2002, 58, 8299; (c) Hirama, M.; Kato, Y.; Seki, Ch.; Matsuyama, H.; Oshikiri, N.; Iyoda, M. Chem. Lett. 2008, 37, 924.

18. (a) Sauer, J.; Sustmann, R. Angew. Chem. Int. Ed. Engl. 1980, 19, 779. (b) Pindur, U.; Lutz, G.; Otto, Ch. Chem. Rev. 1993, 93, 741.

19. (a) Knaus, E. E.; Pasutto, F. M.; Giam, C. S. J. Heterocycl. Chem. 1974, 11, 843. (b) Knaus, E. E.; Pasutto, F. M.; Giam, C. S.; Swinyard, E. A. J. Heterocycl. Chem. 1976, 13, 481. (c) Kane, V. V.; Werblood, H.; Levine, S. D. J. Heterocycl. Chem. 1976, 13, 673. (d) Knaus, E. 
E.; Redda, K. Can. J. Chem. 1977, 55, 1788. (e) Shusherina, N. P.; Said, M.; Likhomanova, T. I. Zh. Org. Khim. 1978, 14, 841. (f) Gazzaeva, R. A.; Drebenkowa, L. V.; Likhomanova, T. I.; Zyk, N. V. Chem. Heterocycl. Comp. 1997, 33, 596.

20. Levine, S.; Kane, V. V. U. S. Patent 4057 547, 1977; Chem. Abstr. 1977, 87, 152222h.

21. (a) Tori, K; Hata, Y.; Muneyuki, R.; Takano, Y.; Tsuji, T.; Tanida, H. Can. J. Chem. 1964, 42, 926. (b) Tomisawa, H.; Fujita, R.; Noguchi, K.; Hongo, H. Chem. Pharm. Bull. 1970, 18,941 .

22. Haukaas, M. H.; O’Doherty, G. A. Org. Lett. 2002, 4, 1771.

23. Bondi, A. J. Phys. Chem. 1964, 68, 441.

24. Clement, R. A. J. Org. Chem. 1960, 25, 1724.

25. In vitro cytotoxic activity was tested according to the procedure described in: Bracht, K.; Boubakari, Grünert, R.; Bednarski, P. J. Anticancer Drugs 2006, 17, 41.

26. (a) Brum-Bousquet, M.; Mitkau, S.; Skaltsounis, A.-L.; Tillequin, F.; Koch, M. Planta Med. 1988, 54, 470. (b) Thi Mai, H. D.; Gaslonde, T.; Michel, S.; Tillequin, F.; Koch, M.; Bongui, J. B.; Elmori, A.; Seguin, E.; Pfeiffer, B.; Renard, P.; David-Cordonnier, M. H.; Laine, W.; Bailly, C.; Kraus-Berthier, L.; Léonce, S.; Hickman, J. A.; Pierré, A. J. Med. Chem. 2003, 46, 3072.

27. Triani, A.; Bellasio, E. J. Heterocycl. Chem. 1974, 11, 257.

28. Myers, A. G.; Zheng, B.; Movassaghi, M. J. Org. Chem. 1997, 62, 7507.

29. Sheldrick, G. M. Acta Cryst. 2008, A64, 112. 